
\title{
25 Research Suare \\ Modeling and Validation of Diamagnetic Rotor Levitated by Permanent Magnetics
}

\section{Yuanping Xu ( $\nabla$ ypxu@nuaa.edu.cn )}

Nanjing University of Aeronautics and Astronautics https://orcid.org/0000-0002-5264-2932

Yue Zhang

Nanjing University of Aeronautics and Astronautics

Jin Zhou

Nanjing University of Aeronautics and Astronautics

Chaowu Jin

Nanjing University of Aeronautics and Astronautics

\section{Original Article}

Keywords: diamagnetic levitation, magnetic levitation, rotor, modeling, validation, stability

Posted Date: November 13th, 2020

DOl: https://doi.org/10.21203/rs.3.rs-102581/v1

License: (9) This work is licensed under a Creative Commons Attribution 4.0 International License. Read Full License 


\title{
Modeling and Validation of Diamagnetic Rotor Levitated by Permanent
}

\author{
Magnetics
}

\author{
Yuanping Xu, Yue Zhang, Jin Zhou*, Chaowu Jin \\ College of Mechanical and Electrical Engineering, Nanjing University of Aeronautics and Astronautics, Nanjing \\ 210016, China.
}

\begin{abstract}
Featured as an innovative powerless and low stiffness suspension approach, diamagnetic levitation technique has trigged intensive interest because of its potential applicability in miniaturized mechanical system. The foundation of diamagnetic levitation system lies in mathematical modeling, which is essential for operating performance optimization and stability predication. However, to date, few literatures concerning the systematic mathematical modeling has been reported. Herein, in this work, a systematic mathematic model for disc-shaped diamagnetically levitated rotor on permanent magnets array is proposed. Based on the proposed model, the magnetic field distribution characteristics, diamagnetic levitation force characteristics (i.e. levitation height and stiffness) expressions and the optimized theorical conditions for realizing stable levitation are achieved. Experiments are also performed to dig out the relationship between levitation height and levitation force. Moreover, the operating stability of rotor under different conditions is evaluated. The experimental results are well in accordance with the theorical predications, confirming the feasibility of this mathematic model.
\end{abstract}

\section{Keywords}

diamagnetic levitation, magnetic levitation, rotor, modeling, validation, stability.

\section{Introduction}

Magnetic levitation technology has been increasingly applied in industrial areas such as bearings, sensors, and magnetic transportation systems because of the distinguished features of avoiding mechanical wear and lubrication [1-7]. Generally, the friction levitation for most of magnetic levitation systems is achieved by employing active feedbackcontrolled electromagnetics. However, the employment of feedback-controlled electromagnetics is always power consuming and requires complicated controller design. Moreover, given that leakage flux would dramatically increase with scale downsizing, it poses an obstacle to realizing miniaturization of mechanical system with the utilization of feedback-controlled electromagnetics [8]. In this regard, the recently developed passive levitated suspension represented by diamagnetic levitation has emerged as an alternative suspension approach for mechanical system, particularly those require low power consumption and small size.

Diamagnetic levitation refers to the free levitation of diamagnetic object in magnetic field. Typical diamagnetic materials include water, bismuth and graphite [9]. With the absence of control system, the suspension structure of diamagnetic levitation system is simple [10-11]. Meanwhile, without the need of energy supply, both statically and dynamically stable diamagnetic levitation can be easily achieved at room temperature. These features make diamagnetic levitation quite appealing for modern micro mechanical system such as microscale separation or manipulation [12] and micro rotor bearing system [13-20]. Additionally, the inherent characterization of low stiffness in diamagnetic levitation system permits its potential application in high-sensitivity sensor [21-24] and ultra-low frequency energy harvesters [2526].

For instance, Liu et al. [15-16] invented a diamagnetically levitated variable-capacitance micro motor and successfully realized the continuous rotation operation of motor. Bleuler et al. [17] demonstrated the rotation of a diamagnetically

\footnotetext{
* Corresponding author. Tel.: +862584892905.

E-mail addresses: ypxu@nuaa.edu.cn,zhj@nuaa.edu.cn
} 
suspended rotor by an electrostatic glass motor. Su et al. [18] reported a micromachined rotor wherein highly oriented pyrolytic graphite was diamagnetically levitated by magnetics and rotated by gas flow. Our previous work [19-20] also realized a rotating diamagnetically levitating rotor system driven by electrostatic field with a maximum rotation speed of $300 \mathrm{rpm}$.

Besides the effort in designing specific micro mechanical system rooting in diamagnetic levitation mechanism, attempt has also been made to construct theoretical and empirical models of diamagnetic levitation system for design and performance optimization. For instance, Chow et al. [27] theoretically analyzed the shape effect of magnetic sources formed by standard coil and ring magnet elements on vertical diamagnetic levitation. Pasquale et al. [28] presented a diamagnetic suspension model based on finite elements software and validated it experimentally. Cansiz et al. [13] developed a unique numerical calculation method to analyze the static and dynamic characterizations of a diamagnetic bearing. Chen et al. [14] developed a numerical model to analyze the performance and stability of diamagnetic bearings for micro-scale applications.

These proposed models and analysis greatly consolidate the theorical foundation of diamagnetic levitation. However, to date, the systematic mathematical modeling of diamagnetic levitation has rarely been reported. Herein, in this work, a systematic mathematical model for disc-shaped diamagnetically levitated rotor on permanent magnets array is proposed and developed. Based on the proposed mathematical model, the magnetic field distribution characteristics, diamagnetic levitation force characteristics (i.e. levitation height and stiffness) expressions and the optimized theorical conditions for realizing stable levitation are achieved. Experiments are also conducted to dig out the relationship between levitation height and levitation force. Furthermore, the operating stability of rotor under different conditions is evaluated. The experimental results are well in accordance with the theorical predications, confirming the feasibility of this mathematic model.

The remainder of the paper is organized as follows. Section 2 describes the diamagnetic levitation test rig. Section 3 presents the modeling and analyzation of magnetic field generated by magnets array. Section 4 describes the modeling and analyzation of diamagnetic force. Experimental validation and discussion are shown in Section 5 and conclusions are drawn in Section 6.

\section{Description of the Diamagnetic Levitation Test Rig}

In our previous work, we developed a diamagnetic contactless suspension rotor driven by electrostatic glass motor [1920]. Here, a more compact test rig consisting of rotor, electrostatic glass motor and array of permanent magnets is designed. The disc-shaped rotor $(\phi 10.38 \mathrm{~mm})$ is comprised of pyrolytic graphite and borosilicate glass (see Fig.1a). The thickness of pyrolytic graphite and borosilicate glass are $500 \mu \mathrm{m}$ and $120 \mu \mathrm{m}$, respectively.

For the array of permanent magnets, a concentric ring-shaped magnet $(\phi 18.68 \times \phi 7.96 \times 12 \mathrm{~mm})$ encircling a cylinder magnet $(\phi 7.94 \times 12 \mathrm{~mm})$ with opposite axial magnetization pattern is adopted, as manifested in Fig. 1b. The diamagnetic rotor is passively and stably levitated at the room temperature by the diamagnetic force between magnets and rotor. The levitation gap between the rotor and the magnets is $0.65 \mathrm{~mm}$. To ensure the rotating function, an electrostatic glass motor driven system is designed. The stator of electrostatic glass motor is fabricated using a flexible printed circuit board (PCB) film (thickness $100 \mu \mathrm{m}$ ) with 12 poles (36 electrodes), which can be placed at the gap between magnets and rotor.
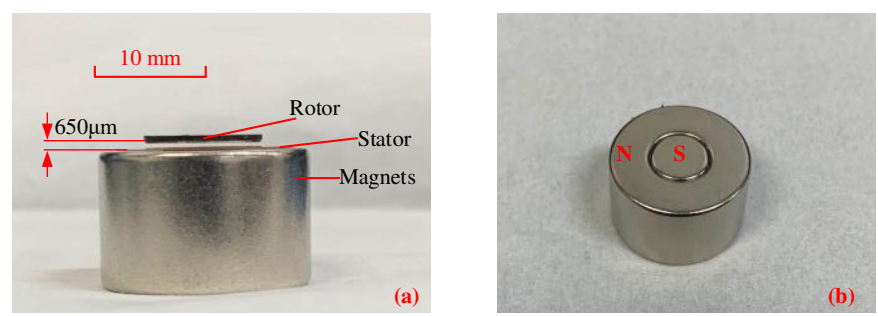

Fig. 1 Diamagnetic levitation test rig, (a) diamagnetic rotor levitated by permanent magnets array, (b) the array of permanent magnets. 
Noted here that the proposed model and corresponding analysis are directed for statically conditions. Considering this, the borosilicate glass of rotor can be eliminated as no electrostatic force is required for rotor rotating. Thus, in the following proposed model and experimental validation, the rotor is only constituted by pyrolytic graphite and the measured levitation height between rotor and magnet is $0.9 \mathrm{~mm}$.

\section{Modeling of Magnetic Field Generated by Magnetic Array}

\subsection{Magnetic Feld Generated by Cylinder Permanent Magnet}

First, the current model is adopted to analyze permanent magnets. According to this model, the magnet is reduced to a distribution of equivalent current. This is then input into the magnetostatic field equations as a source term, and the field is obtained using standard methods for steady currents. Mathematically, a magnet with magnetization $\mathbf{M}$ is equivalent to the following current distribution:

a volume current density

$$
\mathbf{J}_{m}=\nabla \times \mathbf{M}
$$

a surface current density

$$
\mathbf{j}_{m}=\mathbf{M} \times \hat{\mathbf{n}}
$$

where $\hat{\mathbf{n}}$ is the outward unit normal to the magnet surface.

For a better analysis, the cylindrical coordinate is adopted here considering the cylindrical geometry of magnets. The unit vectors of cylindrical coordinates $r, \phi$, and $z$ are $\hat{\mathbf{r}}, \hat{\phi}$, and $\hat{\mathbf{z}}$, respectively. As shown in Fig. 2 , the polarization direction of cylindrical magnet is along its axis. Thus, the volume current density $\mathbf{J}_{m}=\nabla \times \mathbf{M}=\nabla \times M_{s}$ is zero wherein $M_{\mathrm{s}}$ represents the magnetization.
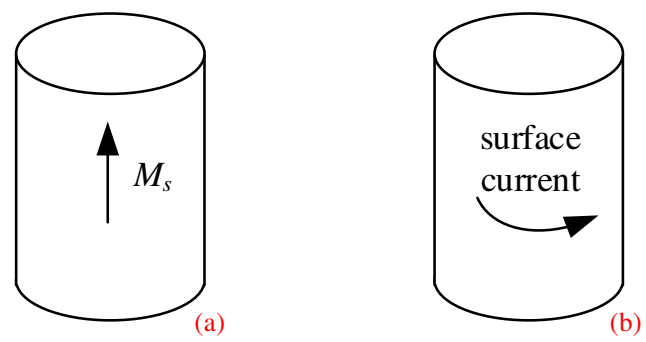

Fig. 2 Cylindrical magnet, (a) polarized along its axis, (b) equivalent surface current.

As for the surface current density, given that top and bottom surface normals are either parallel or antiparallel with the magnetization, $\mathbf{j}_{m}=0$ on the top and bottom surfaces. Consequently, we only consider the outer surface here:

$$
\text { outer surface }=\left\{\begin{array}{c}
r^{\prime}=R \\
0 \leq \phi^{\prime} \leq 2 \pi \\
z_{1} \leq z^{\prime} \leq z_{2}
\end{array}\right.
$$

where $R$ is the outer radius of cylindrical magnet surface, $z_{1}$ and $z_{2}$ denote the axial position of the bottom and top of the cylindrical magnet surface.

The unit normal for the out surface is

$$
\hat{\mathbf{n}}=\hat{\mathbf{r}}
$$

Therefore, the surface current density is 


$$
\mathbf{j}_{m}=\mathbf{M} \times \hat{\mathbf{n}}=M_{s} \hat{\boldsymbol{\phi}}
$$

According to the current model, the vector potential A generated by magnets is [29]

$$
\mathbf{A}(x)=\frac{\mu_{0}}{4 \pi} \int_{v} \frac{\mathbf{J}_{m}\left(x^{\prime}\right)}{\left|x-x^{\prime}\right|} d v^{\prime}+\frac{\mu_{0}}{4 \pi} \tilde{\mathbf{N}} \frac{\mathbf{j}_{m}\left(x^{\prime}\right)}{\left|x-x^{\prime}\right|} d s^{\prime}
$$

where $\mu_{0}$ is the magnetic permeability of vacuum, $x$ is an observation point, and $x^{\prime}$ is a source point. $\left|x-x^{\prime}\right|$ presents the distance between these two points, which is given in the cylindrical coordinate,

$$
\left|x-x^{\prime}\right|=\sqrt{r^{2}+r^{\prime 2}-2 r r^{\prime} \cos \left(\phi-\phi^{\prime}\right)+\left(z-z^{\prime}\right)^{2}}
$$

Since the volume current density $\mathbf{J}_{m}=0$, Thus, (6) can be written

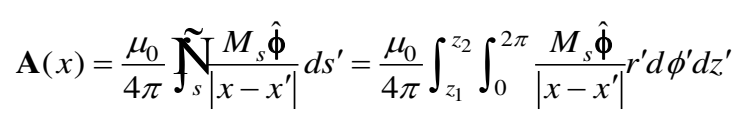

The unit vectors $\hat{\mathbf{x}}$ and $\hat{\mathbf{y}}$ for Cartesian coordinates $x, y$ have the following relationship with cylindrical coordinate unit vectors:

$$
\begin{aligned}
& \hat{\boldsymbol{\phi}}=-\sin (\phi) \hat{\mathbf{x}}+\cos (\phi) \hat{\mathbf{y}} \\
& \hat{\mathbf{r}}=\cos (\phi) \hat{\mathbf{x}}+\sin (\phi) \hat{\mathbf{y}}
\end{aligned}
$$

Substitute (9a) into (8) and obtain

$$
\mathbf{A}(x)=\frac{\mu_{0} M_{s}}{4 \pi} \int_{z_{1}}^{z_{2}} \int_{0}^{2 \pi} \frac{-\sin \left(\phi^{\prime}\right) \hat{\mathbf{x}}+\cos \left(\phi^{\prime}\right) \hat{\mathbf{y}}}{\left|x-x^{\prime}\right|} r^{\prime} d \phi^{\prime} d z^{\prime}
$$

Since the potential $\mathbf{A}(x)$ generated by this cylindrical magnet has no z-component, it can be written in terms of cylindrical coordinates by computing the projections

$$
A_{r}(x)=\mathbf{A}(x) \cdot \hat{\mathbf{r}}
$$

and

$$
A_{\phi}(x)=\mathbf{A}(x) \cdot \hat{\boldsymbol{\phi}}
$$

This gives

$$
A_{r}(x)=\frac{\mu_{0} M_{s}}{4 \pi} \int_{z_{1}}^{z_{2}} \int_{0}^{2 \pi} \frac{\sin \left(\phi-\phi^{\prime}\right)}{\left|x-x^{\prime}\right|} r^{\prime} d \phi^{\prime} d z^{\prime}
$$

and

$$
A_{\phi}(x)=\frac{\mu_{0} M_{s}}{4 \pi} \int_{z_{1}}^{z_{2}} \int_{0}^{2 \pi} \frac{\cos \left(\phi-\phi^{\prime}\right)}{\left|x-x^{\prime}\right|} r^{\prime} d \phi^{\prime} d z^{\prime}
$$

The magnetic flux density $\mathbf{B}$ and vector potential $\mathbf{A}$ are linked by following relation

$$
\mathbf{B}=\nabla \times \mathbf{A}
$$

Therefore, the (15) could be written as 


$$
\mathbf{B}=\left(\frac{1}{r} \frac{\partial A_{z}}{\partial \varphi}-\frac{\partial A_{\varphi}}{\partial z}\right) \hat{\mathbf{r}}+\left(\frac{\partial A_{r}}{\partial z}-\frac{\partial A_{z}}{\partial r}\right) \hat{\boldsymbol{\phi}}+\frac{1}{r}\left(\frac{\partial\left(r A_{\varphi}\right)}{\partial r}-\frac{\partial A_{r}}{\partial \varphi}\right) \hat{\mathbf{z}}
$$

Therefore, the radial and axial field component are given by

$$
\begin{gathered}
B_{r}(x)=-\frac{\partial}{\partial z} A_{\phi}(x) \\
B_{z}(x)=\frac{1}{r}\left(\frac{\partial}{\partial r}\left(r A_{\varphi}(x)\right)-\frac{\partial}{\partial \varphi} A_{r}(x)\right) \\
B_{\varphi}(x)=\frac{\partial}{\partial z} A_{r}(x)
\end{gathered}
$$

Substituting (13) and (14) into (17) yields

$$
\begin{gathered}
B_{r}(r, \phi, z)=\frac{\mu_{0} M_{s}}{4 \pi} \int_{z_{1}}^{z_{2}} \int_{0}^{2 \pi} \frac{\cos \left(\phi-\phi^{\prime}\right)\left(z-z^{\prime}\right)}{\left(r^{2}+r^{\prime 2}-2 r r^{\prime} \cos \left(\phi-\phi^{\prime}\right)+\left(z-z^{\prime}\right)^{2}\right)^{3 / 2}} r^{\prime} d \phi^{\prime} d z^{\prime} \\
B_{z}(r, \phi, z)=\frac{\mu_{0} M_{s}}{4 \pi} \int_{z_{1}}^{z_{2}} \int_{0}^{2 \pi} \frac{-\left(r \cos \left(\phi-\phi^{\prime}\right)-r^{\prime}\right)}{\left(r^{2}+r^{\prime 2}-2 r r^{\prime} \cos \left(\phi-\phi^{\prime}\right)+\left(z-z^{\prime}\right)^{2}\right)^{3 / 2}} r^{\prime} d \phi^{\prime} d z^{\prime} \\
B_{\varphi}(r, \phi, z)=0
\end{gathered}
$$

\subsection{Magnetic Field Generated by the Array of Permanent Magnets}

The array of permanent magnets for diamagnetic levitation system employed here is a concentric ring-shaped magnet encircling a cylinder magnet with opposite axial magnetization pattern. Fig. 3 gives a brief illustration of array structure in permanent magnets.

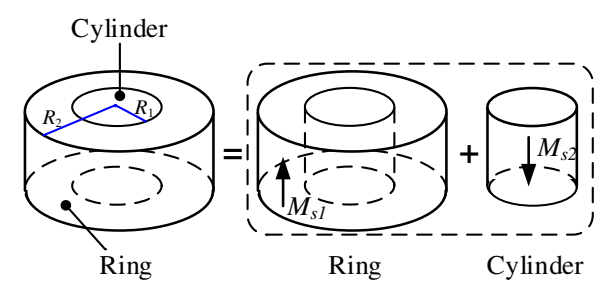

Fig. 3 The array of permanent magnets structure.

For the ring-shaped magnet, there are two remaining surfaces to be considered,

$$
\text { inner surface }=\left\{\begin{array}{c}
r^{\prime}=R_{1} \\
0 \leq \phi^{\prime} \leq 2 \pi \\
z_{1} \leq z^{\prime} \leq z_{2}
\end{array}\right.
$$

and

$$
\text { outer surface }=\left\{\begin{array}{c}
r^{\prime}=R_{2} \\
0 \leq \phi^{\prime} \leq 2 \pi \\
z_{1} \leq z^{\prime} \leq z_{2}
\end{array}\right.
$$

The unit normals for these surfaces are

$$
\hat{\mathbf{n}}= \begin{cases}-\hat{\mathbf{r}} & (\text { inner } \text { surface }) \\ \hat{\mathbf{r}} & (\text { outer } \text { surface })\end{cases}
$$


Therefore, the surface current densities are

$$
\mathbf{j}_{m}= \begin{cases}-M_{s 1} \hat{\boldsymbol{\phi}} & \text { (inner surface) } \\ M_{s 1} \hat{\boldsymbol{\phi}} & \text { (outer surface) }\end{cases}
$$

According to (21), the ring-shaped magnet is anticipated to be equivalent to two cylinder magnets assembled with opposite axial magnetization pattern, as illustrated in Fig.4. Therefore, the arrayed permanent magnets can be equivalent to three cylinder magnets assembled with different axial magnetization pattern (see Fig. 5).

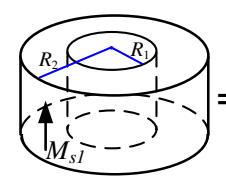

Ring

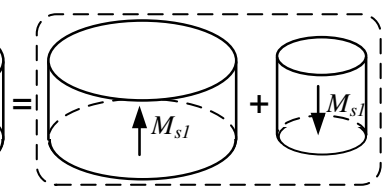

Cylinder
Cylinder

Fig. 4 Ring-shaped magnet equivalence.

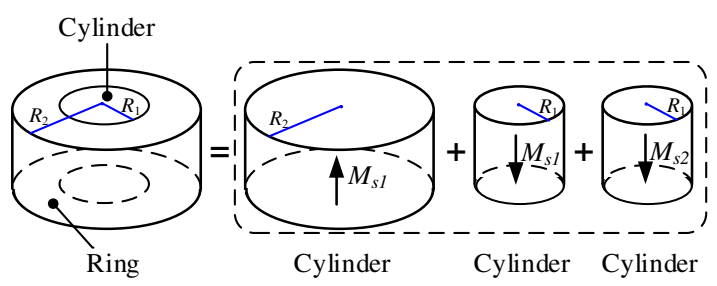

Fig. 5 The array of permanent magnets equivalence.

Therefore, the magnetic flux density $\mathbf{B}$ provided by the array of permanent magnets can be written as

$$
\mathbf{B}=\mathbf{B}_{R 2}+\mathbf{B}_{R 11}+\mathbf{B}_{R 12}
$$

where $\mathbf{B}_{R 2}, \mathbf{B}_{R 11}$, and $\mathbf{B}_{R 12}$ are the magnetic flux density generated by each cylinder magnet. Similarly, the radius $B_{r}$ and axial field component $B_{z}$ generated by the array of permanent magnets could be obtained. Parameters for the magnets array

TABLE I

Parameters for the magnets array

\begin{tabular}{lll}
\hline \hline Symbol & \multicolumn{1}{c}{ Quantity } & \multicolumn{1}{c}{ Values } \\
\hline$z_{1}$ & bottom magnet surface position & $0 \mathrm{~mm}$ \\
$z_{2}$ & top magnet surface position & $12 \mathrm{~mm}$ \\
$M_{s 1}$ & magnetization for inner cylinder magnet & $9.66 \times 10^{5} \mathrm{~A} / \mathrm{m}$ \\
$M_{s 2}$ & magnetization for outer ring magnet & $10.07 \times 10^{6} \mathrm{~A} / \mathrm{m}$ \\
\hline \hline
\end{tabular}

Table I lists the parameters for the magnets array. Based on the deduction mentioned above, we can calculate the magnetic flux density generated by the array of permanent magnets. Shown in Fig. 6 is the obtained magnetic flux density distribution in the plane parallel the top surface of permanent magnets wherein the distance between plane and top surface is fixed to $0.9 \mathrm{~mm}$. From Fig.6, it can be seen that radial component of magnetic flux density $\mathbf{B}_{r}$ first exhibits an up-trend with increasing radial distance from the magnets center and reaches the maximum at the point which is close to the interface between the ring-shaped and cylinder magnets. Afterwards, the magnitude of $\mathbf{B}_{r}$ tends to gradually decline to 0 , followed by another up-trend and then down-trend within a radical distance of $10 \mathrm{~mm}$. In the case of axial field component 
of magnetic flux density $\mathbf{B}_{z}$, the changing-trend appears to be opposite with that of $\mathbf{B}_{r}$ within a radical distance of $10 \mathrm{~mm}$. Specifically, the magnitude of $\mathbf{B}_{z}$ first exhibits a down-trend with increasing radical distance and declines to be 0 at the point close to the interface between the ring-shaped and cylinder magnets. It should be noted that the points corresponding to the maximum $\mathbf{B}_{r}$ and minimum $\mathbf{B}_{z}$ do not locate at the interface between ring-shaped and cylinder magnets, which can be explained by the different magnetization of two magnets.

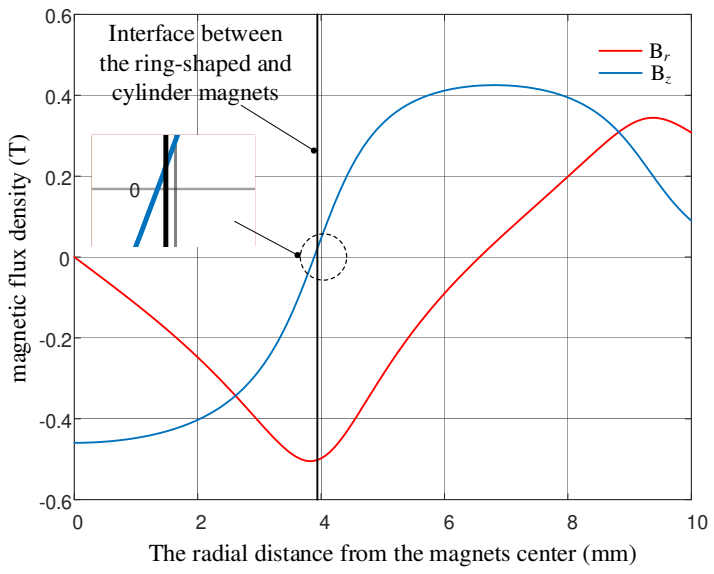

Fig. 6 Theoretical magnetic flux density distribution in the plane parallel the top surface of permanent magnets wherein the distance between plane and top surface is $0.9 \mathrm{~mm}$.

Fig. 7 shows the obtained magnetic flux density $\mathbf{B}_{z}$ and $\mathbf{B}_{r}$ distribution (in the plane parallel the top surface of permanent magnets) versus height from top surface of permanent magnets. Clearly, with increasing height, both the magnitude of $\mathbf{B}_{z}$ and $\mathbf{B}_{r}$ tend to decrease.
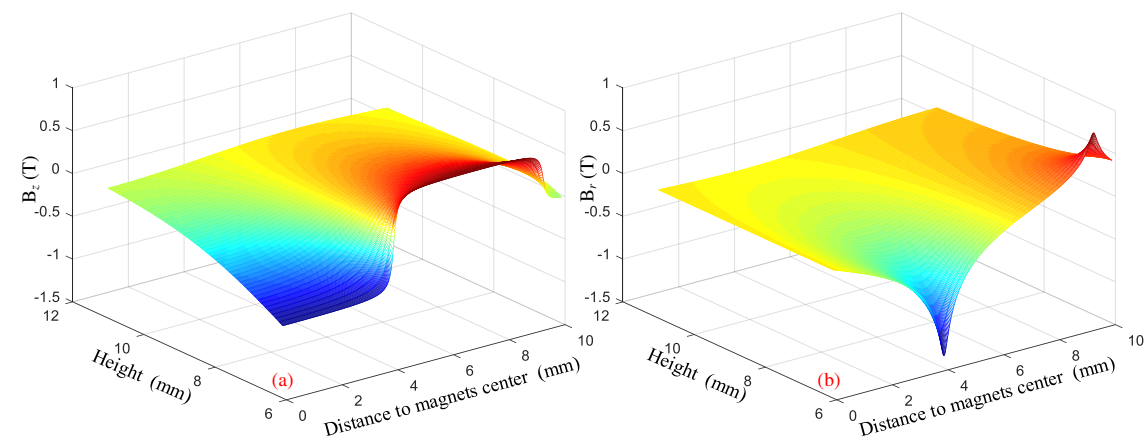

Fig. 7 The height affection for the magnetic flux density generated by the array of permanent magnets, (a) Bz (b) Br.

\section{Modeling of Diamagnetic Force}

\subsection{Diamagnetic Force}

Upon an external magnetic field, the diamagnetic subject can induce an opposite magnetic field. The unit volume potential energy for the diamagnetic material with induced magnetization $\mathbf{M}_{d}$ under the external magnetic flux density $\mathbf{B}$ is

$$
\mathbf{u}=-\mathbf{M}_{d} \cdot \mathbf{B}
$$

Therefore, the elementary diamagnetic force on the unit volume is

$$
\mathbf{f}=-\nabla \mathbf{u}=\mathbf{M}_{d} \cdot \nabla(\mathbf{B})
$$

The magnetic flux density $\mathbf{B}$ and the magnetic field $\mathbf{H}$ are linked by following relation 


$$
\mathbf{H}=\frac{\mathbf{B}}{\mu_{0}}-\mathbf{M}_{d}
$$

The induced magnetization $\mathbf{M}_{d}$ is related to the magnetic flux density $\mathbf{B}$ by the equation

$$
\mathbf{M}_{d}=\chi_{m} \mathbf{H}
$$

where $\chi_{m}$ is the magnetic susceptibility. For the diamagnetic materials pyrolytic graphite, as the $\chi_{\mathrm{m}}$ is very small, given (25-26), the induced magnetization $\mathbf{M}_{d}$ can be expressed and simplified as a function of $\mathbf{B}$,

$$
\mathbf{M}_{d}=\frac{\chi_{m}}{\left(1+\chi_{m}\right) \mu_{0}} \mathbf{B} \approx \frac{\chi_{m}}{\mu_{0}} \mathbf{B}
$$

This simplification implies that the influence of the diamagnetic material on the external magnetic field could be almost neglected. Therefore, the expression of the diamagnetic force per unit volume in function of the magnetic flux density becomes

$$
\mathbf{f}=\frac{\chi_{m}}{\mu_{0}} \mathbf{B} \cdot \nabla(\mathbf{B})
$$

The total magnetic force acting on the diamagnetic body can be obtained by integrating the unit force on the entire volume:

$$
\mathbf{F}=\frac{\chi_{m}}{2 \mu_{0}} \iiint_{V}\left(\nabla\left(\mathbf{B}^{2}\right)\right) \cdot d v
$$

When the magnetic susceptibility $\chi_{m}$ is negative, the reaction force according to (29) provided by the magnetic field will push the diamagnetic body out of the field. However, as the magnetic susceptibility $\chi_{m}$ for most diamagnetic materials at room temperature is very small, the diamagnetic force is relatively weak. Therefore, the diamagnetic levitation is promising for micro mechanical system.

From (29), the diamagnetic force in each orthogonal direction is

$$
\begin{aligned}
& F_{r}=\frac{\chi_{m}}{\mu_{0}} \iiint_{V}\left(B_{r} \frac{\partial B_{r}}{\partial r}\right) \cdot d v=\frac{\chi_{m}}{2 \mu_{0}} \iiint_{V}\left(\frac{\partial\|\mathbf{B}\|^{2}}{\partial r}\right) \cdot d v(30 \mathrm{a}) \\
& F_{z}=\frac{\chi_{m}}{\mu_{0}} \iiint_{V}\left(B_{z} \frac{\partial B_{z}}{\partial z}\right) \cdot d v=\frac{\chi_{m}}{2 \mu_{0}} \iiint_{V}\left(\frac{\partial\|\mathbf{B}\|^{2}}{\partial z}\right) \cdot d v(30 \mathrm{~b})
\end{aligned}
$$

According to Ostrogradsky theorem, the above equations could be rewritten as

$$
\begin{aligned}
& F_{r}=\frac{\chi_{m}}{2 \mu_{0}} \iint_{s}\|\mathbf{B}\|^{2} \hat{\mathbf{r}} \cdot d s \\
& F_{z}=\frac{\chi_{m}}{2 \mu_{0}} \iint_{s}\|\mathbf{B}\|^{2} \hat{\mathbf{z}} \cdot d s
\end{aligned}
$$

For the disc-shaped rotor levitation system, as the theoretical diamagnetic susceptibility of pyrolytic graphite in radial direction is lower than that in axis direction and the thickness of disc-shaped rotor is small, the resultant $\mathrm{F}_{y}$ is small and can be almost neglected. Consequently, the vertical diamagnetic force is mainly responsible for overcoming the gravity force and supporting levitation. Considering that, our primary concern lies in the vertical diamagnetic force property. For the disc-shaped rotor adopted in this paper, (31b) could be simplified as

$$
F_{z}=\frac{\chi_{m}}{2 \mu_{0}} \int_{0}^{r_{d}} 2 B_{z}^{2} \pi r d r
$$


where $r_{\mathrm{d}}$ is the radius of the disc-shaped rotor. The theoretical diamagnetic susceptibility $\chi_{\mathrm{m}}$ of the pyrolytic graphite material is $\chi_{m}=-450 \times 10^{-6}$. However, due to unavoidable defects, it is hard to ensure the good quality of commercial pyrolytic graphite and thus the real $\chi_{\mathrm{m}}$ of pyrolytic graphite is generally lower than that of theoretical value. Additionally, the different processing history and rare materials of pyrolytic graphite may cause variation in the real $\chi_{\mathrm{m}}$. In this work, we find that a $\chi_{\mathrm{m}}$ value of around $\chi_{m}=-320 \times 10^{-6}$ is in accordance with the experimental data. Thus, $\chi_{\mathrm{m}}$ value of $\chi_{m}=-320 \times 10^{-6}$ is adopted for modeling here. According to (32), we calculate the diamagnetic levitation force applied on the rotor at different levitation height and results are presented in Fig.8. As shown, the diamagnetic levitation force and levitation height are nonlinearly related. With the increasing levitation height, the diamagnetic levitation force gradually decreases.

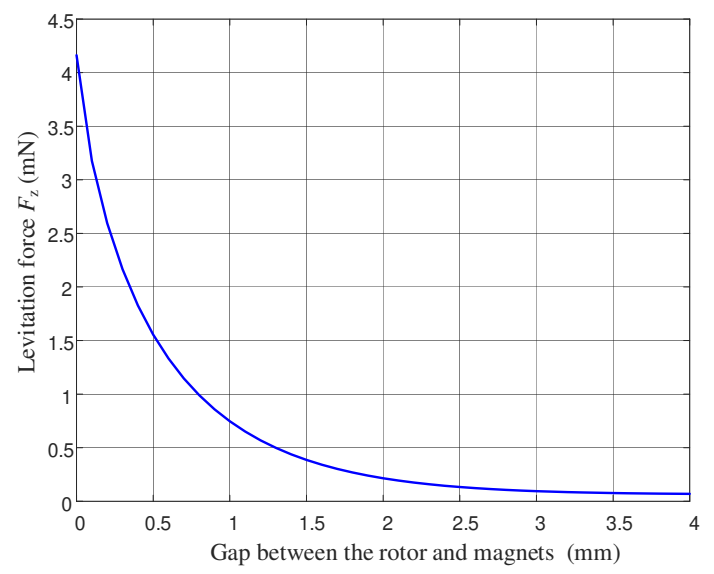

Fig. 8 Theoretical diamagnetic levitation force versus levitation height.

\subsection{Stiffness}

The stiffness is an important parameter for calculating force generation, resonant frequency, and predicating system behavior. The stiffness of the diamagnetic levitation force for diamagnetic rotor at different vertical air gap (levitation height) can be obtained from the derivation of the magnetic force, which is listed in (33).

$$
k_{z z}=-\frac{\partial F_{z}}{\partial z}=-\frac{\chi_{m}}{\mu_{0}} \iint_{s}\left(B_{z} \frac{\partial B_{z}}{\partial z}\right) \cdot d s
$$

With a fixed air gap, the stiffness of the diamagnetic levitation force for the diamagnetic rotor at different radial distance can be obtained from the derivation of (32), which is

$$
k_{z r}=\frac{\partial F_{z}}{\partial r}=\frac{\chi_{m}}{\mu_{0}} B_{z}^{2} \pi r
$$



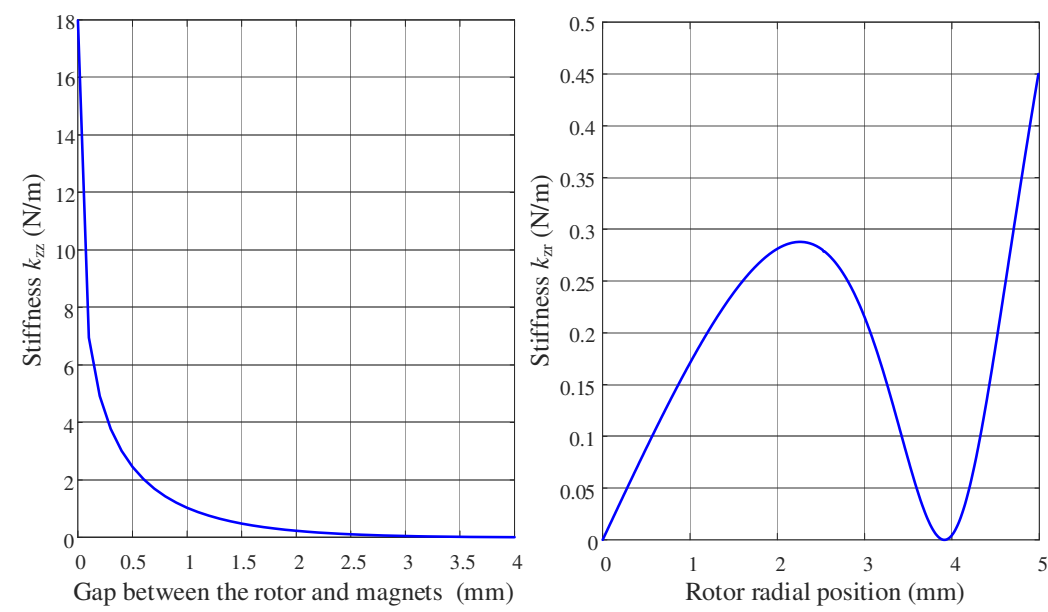

Fig. 9 The stiffness property of the diamagnetic levitation force, (a) at different height, (b) at different rotor radial position with $0.9 \mathrm{~mm}$ height.

Fig.9(a) shows the theoretical stiffness of diamagnetic levitation force versus air gap (levitation height). Clearly, a nonlinear relationship between theoretical stiffness and levitation height can be observed. Specifically, the theoretical stiffness of diamagnetic levitation force sharply decreases first and then slowly declines to zero. Fig. 9(b) describes the theoretical stiffness of diamagnetic levitation force versus rotor radial position with a fixed levitation height of $0.9 \mathrm{~mm}$. It can be seen that with a fixed levitation height, the theoretical stiffness of diamagnetic levitation force first consistently increases with increasing radial distance and reaches the peak. The further increasing radial distance would lead to a decline in theoretical stiffness. Once the radial distance increases around to the point corresponding to the interface between the ring-shaped and cylinder magnets, the theoretical stiffness declines to 0 , followed by a further up-trend.

\subsection{Stability}

Stability is another critical concern for magnetic levitation system. In the diamagnetic levitation system, steady levitation cannot be ensured when diamagnetic levitation force can only overcome gravity. As mentioned above that $F_{y}$ can be almost neglected, and thus the stability of rotor is determined by the torque. Here, the rotor mas center is selected as centroid for torque calculation. For better analysis, the cylindrical coordinates expressed in (32) is transferred into Cartesian coordinate. Assuming that the rotor performs an eccentricity $e$ along the $x$ direction, the torque induced by diamagnetic levitation force can be expressed as (35)

$$
M_{e}=\frac{\chi_{m}}{\mu_{0}} \iint_{s} B_{z}^{2}(x, y)(x-e) d x d x
$$

where

$$
B_{z}(x, y)=\left.B_{z}(r)\right|_{r=\sqrt{x^{2}+y^{2}}}
$$




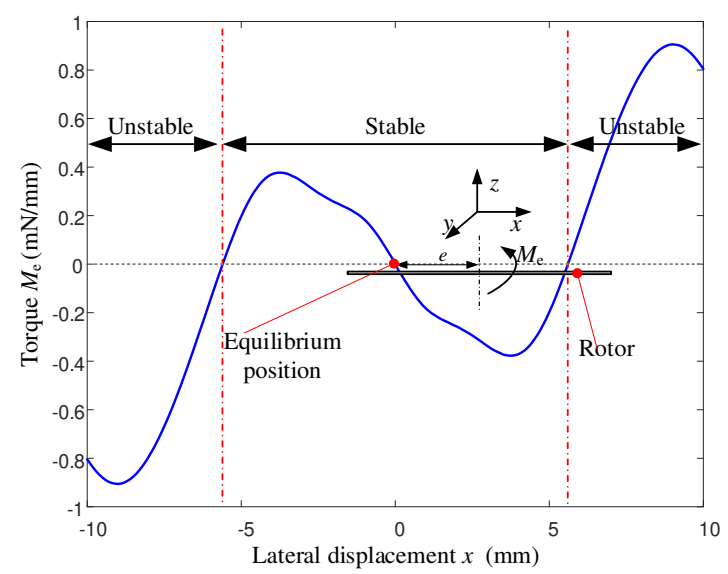

Fig. 10 The torques applied to the rotor considering different lateral displacement (rotor radius is $5.19 \mathrm{~mm}$, levitation height is $0.9 \mathrm{~mm}$ )

Displayed in Fig.10 is the lateral displacement of rotor versus torque obtained from (35) with a fixed rotor radius of $5.19 \mathrm{~mm}$ and levitation height of $0.9 \mathrm{~mm}$. From Fig.10, one can see that when rotor shifts towards the positive $x$ direction and locates at the stable region, it would be subject to an anticlockwise torque in the $y$ direction and then return to the center of permanent magnet. However, once the rotor shifts beyond the stable region, the rotor would be subject to a clockwise torque in the $y$ direction and then drop off from the permanent magnet. Similar phenomenon can be found in the case where rotor shifts towards the negative $x$ direction.

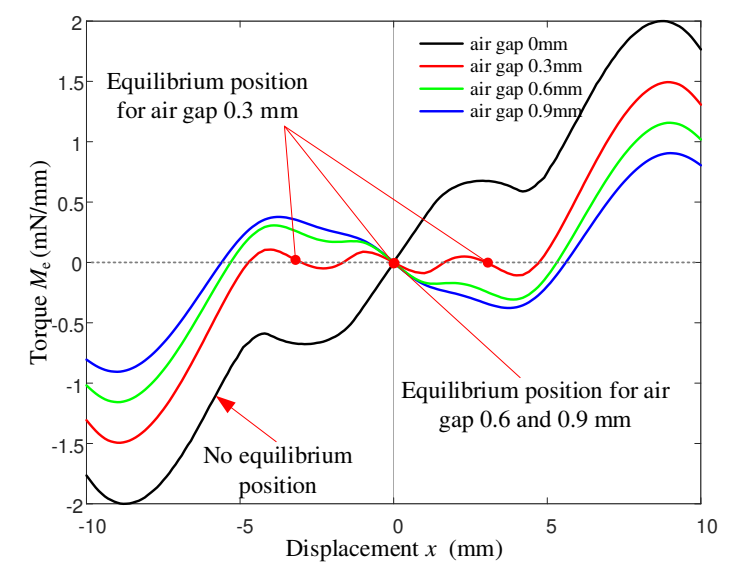

Fig. 11 The lateral displacement of rotor versus torque under different levitation height of $0,0.3,0.6$ and 0.9 mm (rotor radius is $5.19 \mathrm{~mm}$ )

Based on (35), we also analyse the lateral displacement of rotor versus torque under different levitation height and the results are manifested in Fig.11. From Fig.11, it can be concluded that the levitation height can strongly affect the levitating stability. With a levitation height of $0 \mathrm{~mm}$, the rotor is unable to levitate stably. When the levitation height increases to $0.3 \mathrm{~mm}$, the rotor has three equilibrium points wherein it can stably levitate. With the further increase in levitation height, the number of equilibrium point for rotor turns into only one. Moreover, from Fig.11, it can be deduced that increasing levitation height would result in enlarged stable region.

To better illustrate the effect of levitation height on rotor stability, the torque of rotor versus levitation height and lateral displacement is given in Fig.12. To make it clearer, the regions wherein torque is beyond 0 are marked with dots in Fig.12. From Fig.12, one can conclude that number of equilibrium point for rotor varies at different levitation heights and increasing levitation height is advantageous to broaden stable region, which corroborates the analysis derived from Fig.11. 


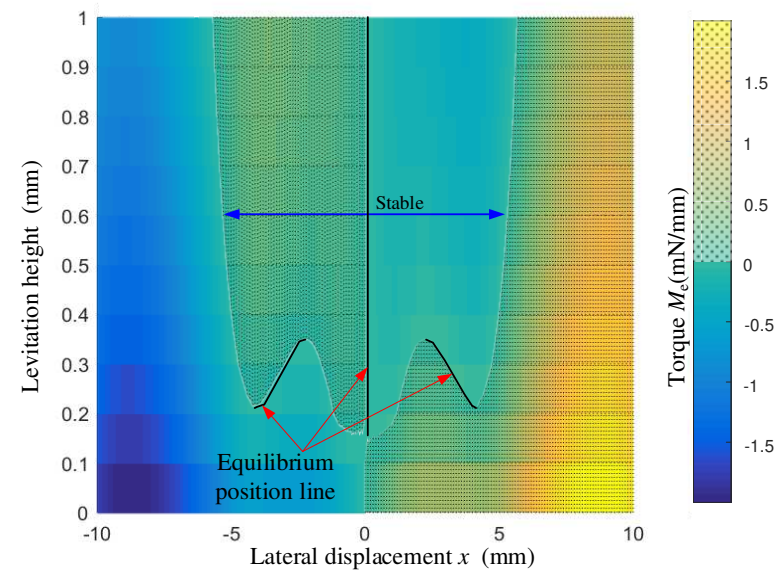

Fig. 12 Torque of rotor versus levitation height and lateral displacement (rotor radius is $5.19 \mathrm{~mm}$ ).

The radius size of rotor is another critical factor determining its levitation stability. To elucidate the effect of rotor size on levitation stability, we analyse the torque of rotor versus rotor radius and rotor lateral displacement based on (35) with a fixed levitation height. It should be mentioned here that for a distinct graphing, the numerical values in z-axis are nondimension processed as described below.

$$
z=M_{e} / m g r
$$

The resultant graph is shown in Fig.13 which gives the torque of rotor versus rotor radius and rotor lateral displacement. Apparently, with increasing rotor radius, the equilibrium line where supports stable levitation would change from the interface between the ring-shaped and cylinder magnets to the magnets center. However, with the further increase in rotor radius, the stable regions for rotor would gradually diminish and even vanish. Thus, it can be concluded that with a fixed levitation, a moderate rotor radius can permit a stable levitation.

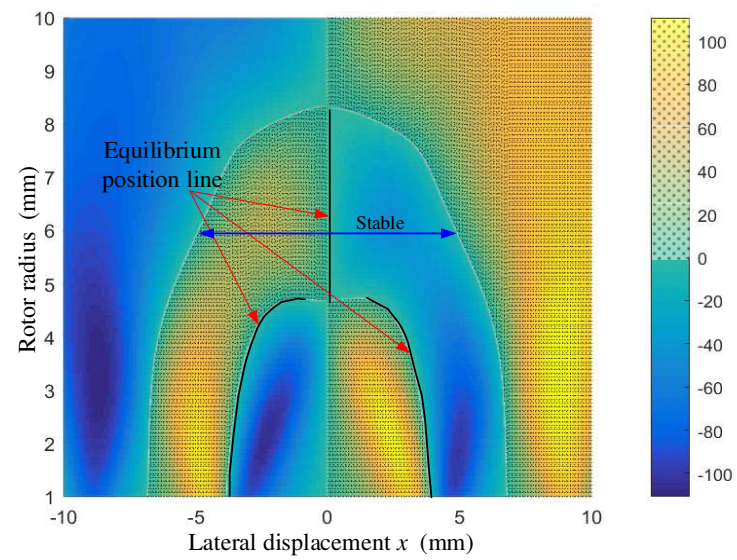

Fig. 13 The torque of rotor versus rotor radius and rotor lateral displacement (levitation height $0.9 \mathrm{~mm}$ )

It worth noting here that the obtained results shown in Fig.13 is achieved with a fixed levitation height of $0.9 \mathrm{~mm}$. In fact, the change in rotor radius would cause the change in weight and area. Consequently, the levitation height may vary. Additionally, in the case where rotor radius is tiny, unstable levitation may occur at the interface between ring-shaped and cylinder magnets. This may be explained by the fact that the corresponding $\mathbf{B}_{z}$ reaches the minimum, which is not large enough to conquer gravity. 


\section{Experimental Verification}

\subsection{Experimental Setup Description}

Illustrated in Fig. 14a is the photography of magnetic field distribution tester, which is employed for the magnetic flux density measurement. The magnets array is placed at the XY linear motor stage and the Teslameter is fixed onto the precision Z-stage. The air gap between the Teslameter and the magnets is fixed to $0.9 \mathrm{~mm}$, which is in accordance with the levitation height of rotor. Considering that the Teslameter can only test the magnetic flux density in vertical direction, we only perform experiments to characterize $\mathbf{B}_{z}$.

Fig. 14b depicts the schematic drawing of set-up for levitation force measurement. As shown, A precision scale is fixed to the base to measure the force. The permanent magnets array is fixed to the stepper motor to modulate the air gap between the magnets and diamagnetic rotor. To eliminate the effect of magnets on precision scale, a plastic rod is used to support diamagnetic rotor and provide enough distance between the magnets and precision scale. The mass of the plastic rotor is $10.0033 \mathrm{~g}$ and the mass of the diamagnetic rotor is $0.0903 \mathrm{~g}$. Stepper motor is set to descend with a distance of 0.1 mm each step and corresponding data is recorded until the data shown in scale turns into stable.
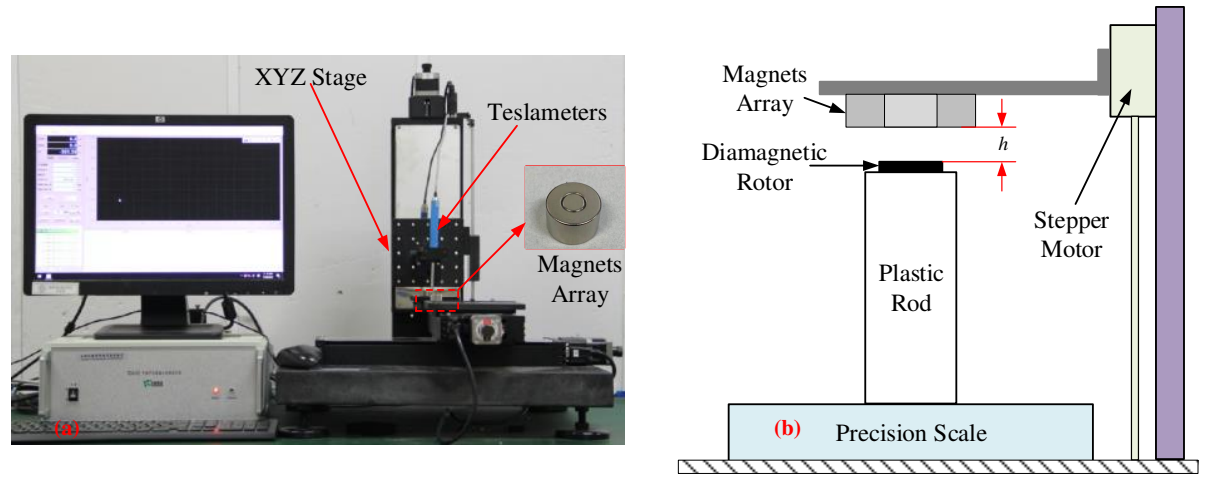

Fig. 14 Experimental setup, (a) magnetic field distribution tester, (b) diamagnetic levitation force measurement schematic diagram.

\subsection{Experimental Results and Discussion}

Fig. 15a presents the comparison of the magnetic flux density $\mathbf{B}_{z}$ obtained from the model and experiment with a fixed levitation height of $0.9 \mathrm{~mm}$. Clearly, the theorical values are well in accordance with the experimental results despite some small errors. The errors may be induced by several factors, such as the insufficient magnetizing of magnets, materials issue of magnets or measurement errors.

Fig. $15 \mathrm{~b}$ is a comparison of diamagnetic force obtained from the model and experiment. Evidently, the theorical diamagnetic force fit quite well with the experimental data, indicting the feasibility and applicability of the proposed model. It also should be mentioned that with a levitation height of $0.9 \mathrm{~mm}$, the diamagnetic force obtained by experiment is $0.881 \mathrm{mN}$, which is comparable with the gravity of rotor $(0.885 \mathrm{mN}, 0.0903 \mathrm{~g})$. This again confirms the of feasibility of model.
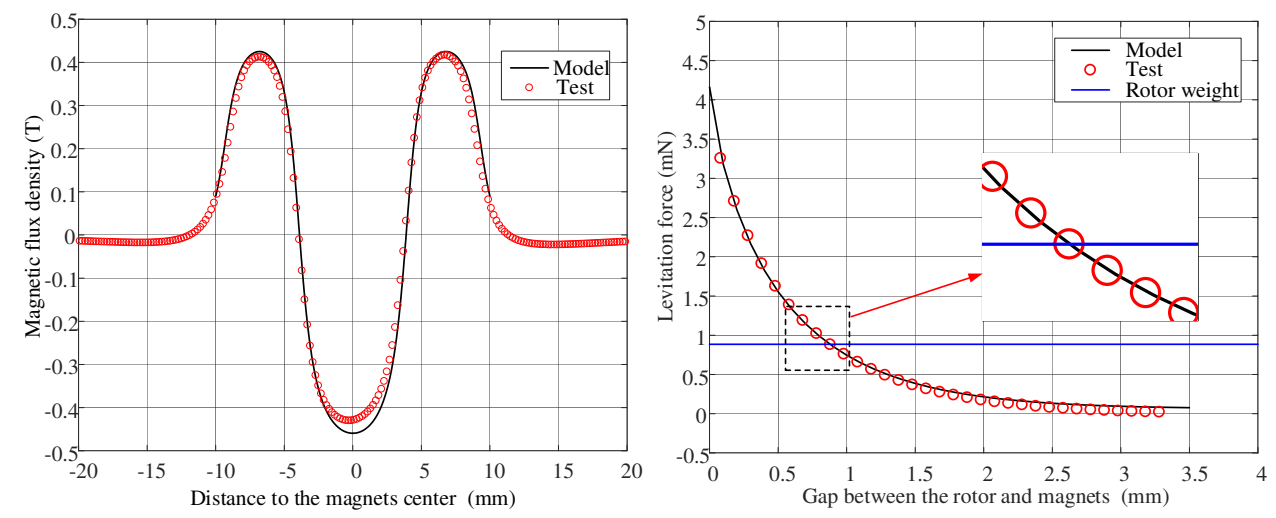
Fig. 15 Comparison from the test and the model, (a) the magnetic flux density Bz, (b) the diamagnetic levitation force.

The levitation stability of rotor with various radius $(5.19,6.25,8.50 \mathrm{~mm})$ are also evaluated. Note here that the pyrolytic graphite with a thickness of $0.5 \mathrm{~mm}$ are acted as rotor in experiments. The results reveal that rotors with radius of 5.19 and $6.25 \mathrm{~mm}$ can realize stable levitation, as evidenced in Fig.16. This corroborates the stability predication mentioned above that a moderate radius can ensure stable levitation (see Fig.13). For the rotor with a radius of $8.5 \mathrm{~mm}$, unstable levitation would occur without the application of external force. This phenomenon also verifies the stability predication that rotor with large radius would undergo unstable levitation.
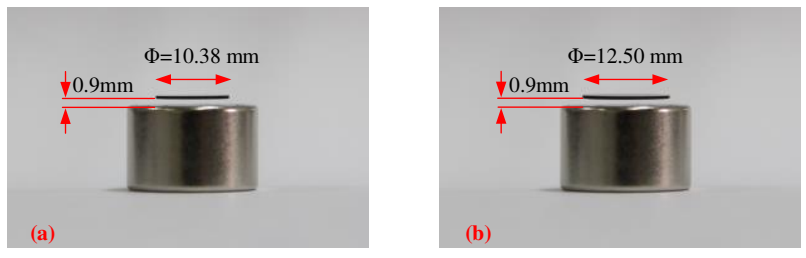

Fig. 16 Levitation stability test, (a) radius $5.19 \mathrm{~mm}$, (b) radius $6.25 \mathrm{~mm}$
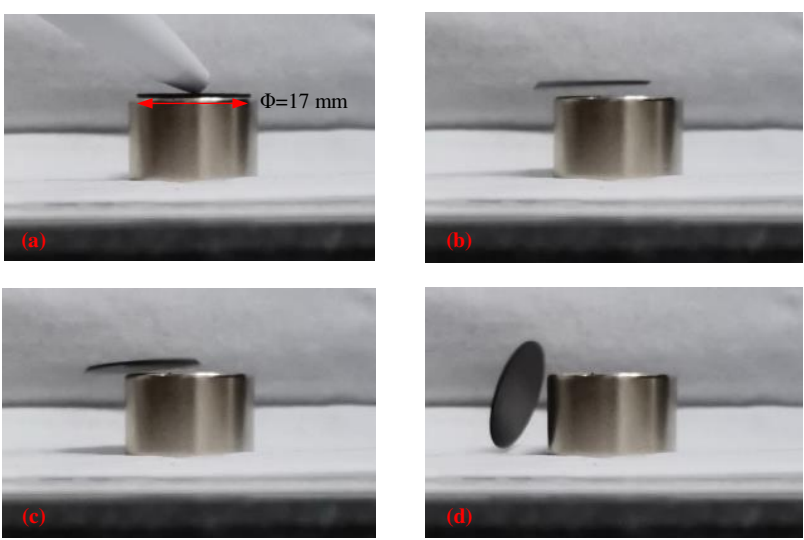

Fig. 17 Levitation stability test for radius $8.50 \mathrm{~mm}$ rotor.

It worth noting that the levitation experiment for rotor with radius of $1.5 \mathrm{~mm}$ has also been attempted. However, it is found that the rotor is unable to fully levitate, which appears to contradict the theorical analysis. However, it can be rationalized by the fact that the $\mathbf{B}_{z}$ of rotor with tiny radius is too weak to conquer gravity. Thus, even the rotor locates at the equilibrium line (interface between the ring-shaped and cylinder magnets) and the toque is 0 , unstable levitation still occurs.

\section{Conclusion}

A systematic mathematical model for disc-shaped diamagnetically levitated rotor on permanent magnets array is proposed and developed in this work. Making use of the proposed mathematical model, the magnetic field distribution characteristics, diamagnetic levitation force characteristics (i.e. levitation height and stiffness) expressions and the optimized theorical conditions for realizing stable levitation are discussed and analyzed. Afterwards, experiments validation is performed. For instance, the relationship between levitation height and levitation force is experimental confirmed. Meanwhile, the operating stability of rotor under different conditions is evaluated. The obtained experimental results are in accordance with the theorical predications, confirming the feasibility of this mathematic model.

\section{Acknowledgment}


The authors would like to thank Mr. Xinxin Guo for photoing Fig.17.

\section{Funding}

This work was supported by the National Natural Science Foundation of China (51905263), Jiangsu Natural Science Youth Fund (BK20190412) and Jiangsu Postdoctoral Research Funding (2019K188).

\section{Competing Interests}

The authors declare no competing financial interests 。

\section{Authors' Contributions}

Yuanping $\mathrm{Xu}$ was in charge of the whole research and wrote the manuscript; Yue Zhang assisted with the system stability analysis and figure drawing. Jin Zhou revised the final manuscript and Chaowu Jin assisted with the magnets manufacturing.

\section{References}

[1] S.-I. Seo, M. K. Lee, S.-J. Kim, and N. Kim, "Robust optimum design of a bearingless rotation motor using the Kriging model,” Int. J. Precis. Eng. Manuf., vol. 12, no. 6, pp. 1043-1050, Dec. 2011, doi: 10.1007/s12541-0110139-z.

[2] S. E. Li, J.-W. Park, J.-W. Lim, and C. Ahn, "Design and control of a passive magnetic levitation carrier system," Int. J. Precis. Eng. Manuf., vol. 16, no. 4, pp. 693-700, Apr. 2015, doi: 10.1007/s12541-015-0092-3.

[3] F. C. Moon, Superconducting Levitation. Wiley, 1995.

[4] H. Zhu, T. J. Teo, and C. K. Pang, "Magnetically Levitated Parallel Actuated Dual-Stage (Maglev-PAD) System for Six-Axis Precision Positioning,” IEEE/ASME Trans. Mechatronics, vol. 24, no. 4, pp. 1829-1838, Aug. 2019, doi: 10.1109/TMECH.2019.2928978.

[5] H. Yaghoubi, "The Most Important Maglev Applications," J. Eng., vol. 2013, pp. 1-19, 2013, doi: $10.1155 / 2013 / 537986$.

[6] K. Wang, X. Ma, Q. Liu, S. Chen, and X. Liu, "Multiphysics Global Design and Experiment of the Electric Machine With a Flexible Rotor Supported by Active Magnetic Bearing," IEEE/ASME Trans. Mechatronics, vol. 24, no. 2, pp. 820-831, Apr. 2019, doi: 10.1109/TMECH.2019.2892392.

[7] G. Li, Z. Lin, P. E. Allaire, and J. Luo, "Modeling of a High Speed Rotor Test Rig With Active Magnetic Bearings," J. Vib. Acoust., vol. 128, no. 3, pp. 269-281, Jun. 2006, doi: 10.1115/1.2172254.

[8] E. H. Maslen and G. Schweitzer, Eds., Magnetic Bearings. Berlin, Heidelberg: Springer Berlin Heidelberg, 2009.

[9] S. A. Peyman, E. Y. Kwan, O. Margarson, A. Iles, and N. Pamme, "Diamagnetic repulsion-A versatile tool for label-free particle handling in microfluidic devices,” J. Chromatogr. A, vol. 1216, no. 52, pp. 9055-9062, Dec. 2009, doi: 10.1016/j.chroma.2009.06.039.

[10]Q. Wang et al., “A diamagnetic levitation based inertial sensor for geophysical application,” Sensors Actuators A Phys., vol. 312, p. 112122, Sep. 2020, doi: 10.1016/j.sna.2020.112122.

[11] S. Palagummi and F. G. Yuan, "An optimal design of a mono-stable vertical diamagnetic levitation based electromagnetic vibration energy harvester," J. Sound Vib., vol. 342, pp. 330-345, Apr. 2015, doi: 10.1016/j.jsv.2014.12.034.

[12]Q.-H. Gao et al., "Label-free manipulation via the magneto-Archimedes effect: fundamentals, methodology and applications," Mater. Horizons, vol. 6, no. 7, pp. 1359-1379, 2019, doi: 10.1039/C8MH01616J.

[13] A. Cansiz and J. R. Hull, "Stable Load-Carrying and Rotational Loss Characteristics of Diamagnetic Bearings,” IEEE Trans. Magn., vol. 40, no. 3, pp. 1636-1641, May 2004, doi: 10.1109/TMAG.2004.827181. 
[14] J.-Y. Chen, J.-B. Zhou, and G. Meng, "Diamagnetic bearings for MEMS: Performance and stability analysis,” Mech. Res. Commun., vol. 35, no. 8, pp. 546-552, Dec. 2008, doi: 10.1016/j.mechrescom.2008.06.008.

[15] W. Liu, W.-Y. Chen, W.-P. Zhang, X.-G. Huang, and Z.-R. Zhang, "Variable-capacitance micromotor with levitated diamagnetic rotor,” Electron. Lett., vol. 44, no. 11, p. 681, 2008, doi: 10.1049/el:20080528.

[16] W. Liu, W. Zhang, and W. Chen, "Simulation analysis and experimental study of the diamagnetically levitated electrostatic micromotor,” J. Magn. Magn. Mater., vol. 492, p. 165634, Dec. 2019, doi: 10.1016/j.jmmm.2019.165634.

[17] R. Moser and H. Bleuler, "Precise positioning using electrostatic glass motor with diamagnetically suspended rotor," IEEE Trans. Appiled Supercond., vol. 12, no. 1, pp. 937-939, Mar. 2002, doi: 10.1109/TASC.2002.1018553.

[18] Y. Su, Z. Xiao, Z. Ye, and K. Takahata, “Micromachined Graphite Rotor Based on Diamagnetic Levitation,” IEEE Electron Device Lett., vol. 36, no. 4, pp. 393-395, Apr. 2015, doi: 10.1109/LED.2015.2399493.

[19] Y. Xu, Q. Cui, R. Kan, H. Bleuler, and J. Zhou, "Realization of a Diamagnetically Levitating Rotor Driven by Electrostatic Field," IEEE/ASME Trans. Mechatronics, vol. 22, no. 5, pp. 2387-2391, Oct. 2017, doi: 10.1109/TMECH.2017.2718102.

[20] Y. Xu, J. Zhou, H. Bleuler, and R. Kan, "Passive diamagnetic contactless suspension rotor with electrostatic glass motor," Micro Nano Lett., vol. 14, no. 10, pp. 1056-1059, Sep. 2019, doi: 10.1049/mnl.2019.0144.

[21]Q. Li, K.-S. Kim, and A. Rydberg, "Lateral force calibration of an atomic force microscope with a diamagnetic levitation spring system,” Rev. Sci. Instrum., vol. 77, no. 6, p. 065105, Jun. 2006, doi: 10.1063/1.2209953.

[22] M. Boukallel, J. Abadie, and E. Piat, "Levitated micro-nano force sensor using diamagnetic materials," in 2003 IEEE International Conference on Robotics and Automation (Cat. No.03CH37422), vol. 3, pp. 3219-3224, doi: 10.1109/ROBOT.2003.1242086.

[23] S. Clara, H. Antlinger, W. Hilber, and B. Jakoby, "Viscosity and density sensor principle based on diamagnetic levitation using pyrolytic graphite," in 2013 IEEE SENSORS, Nov. 2013, pp. 1-4, doi: 10.1109/ICSENS.2013.6688491.

[24] K. Zhang, Y. Su, J. Ding, Q. Gong, and Z. Duan, "Design and Analysis of a Gas Flowmeter Using Diamagnetic Levitation,” IEEE Sens. J., vol. 18, no. 17, pp. 6978-6985, Sep. 2018, doi: 10.1109/JSEN.2018.2853680.

[25]L. Liu and F. G. Yuan, "Nonlinear vibration energy harvester using diamagnetic levitation,” Appl. Phys. Lett., vol. 98, no. 20, p. 203507, May 2011, doi: 10.1063/1.3583675.

[26] S. Palagummi and F. G. Yuan, "A bi-stable horizontal diamagnetic levitation based low frequency vibration energy harvester," Sensors Actuators A Phys., vol. 279, pp. 743-752, Aug. 2018, doi: 10.1016/j.sna.2018.07.001.

[27] T. C. S. Chow, P. L. Wong, and K. P. Liu, "Shape Effect of Magnetic Source on Stabilizing Range of Vertical Diamagnetic Levitation,” IEEE Trans. Magn., vol. 48, no. 1, pp. 26-30, Jan. 2012, doi: 10.1109/TMAG.2011.2168824.

[28] G. De Pasquale, S. Iamoni, and A. Somà, "3D numerical modeling and experimental validation of diamagnetic levitating suspension in the static field," Int. J. Mech. Sci., vol. 68, pp. 56-66, Mar. 2013, doi: 10.1016/j.ijmecsci.2012.12.018.

[29]E. P. Furlani, Permanent Magnet and Electromechanical Devices. Elsevier, 2001. 
Figures
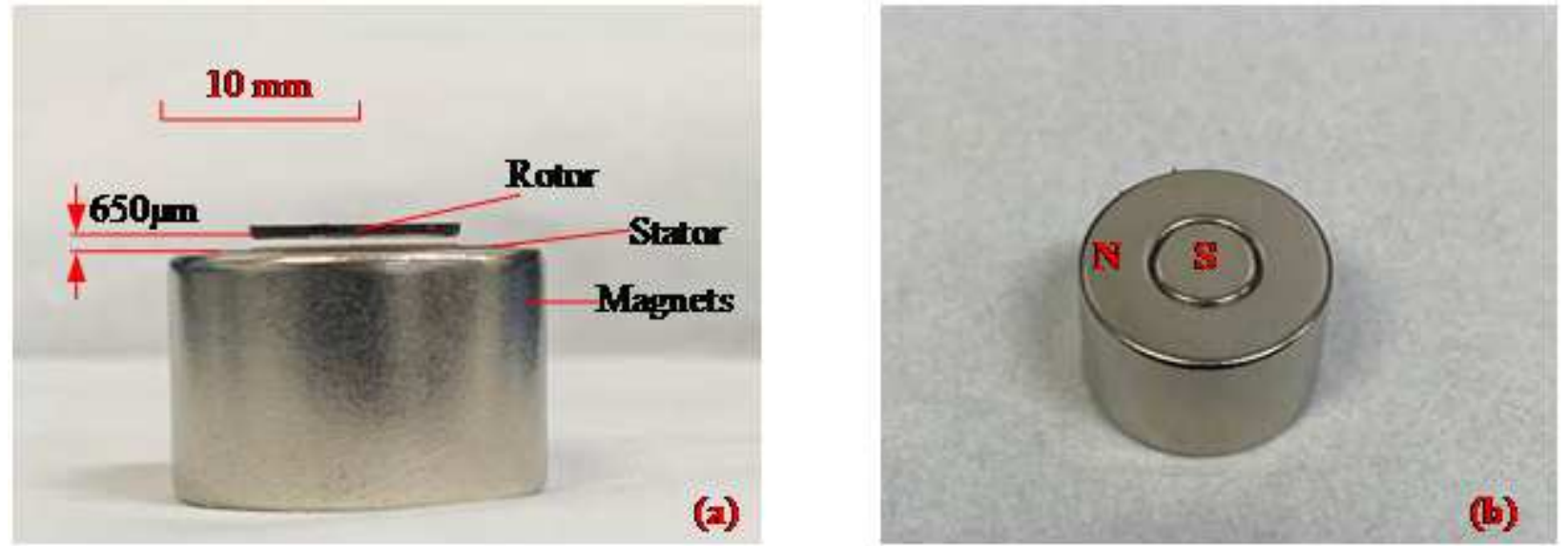

Figure 1

Diamagnetic levitation test rig, (a) diamagnetic rotor levitated by permanent magnets array, (b) the array of permanent magnets.
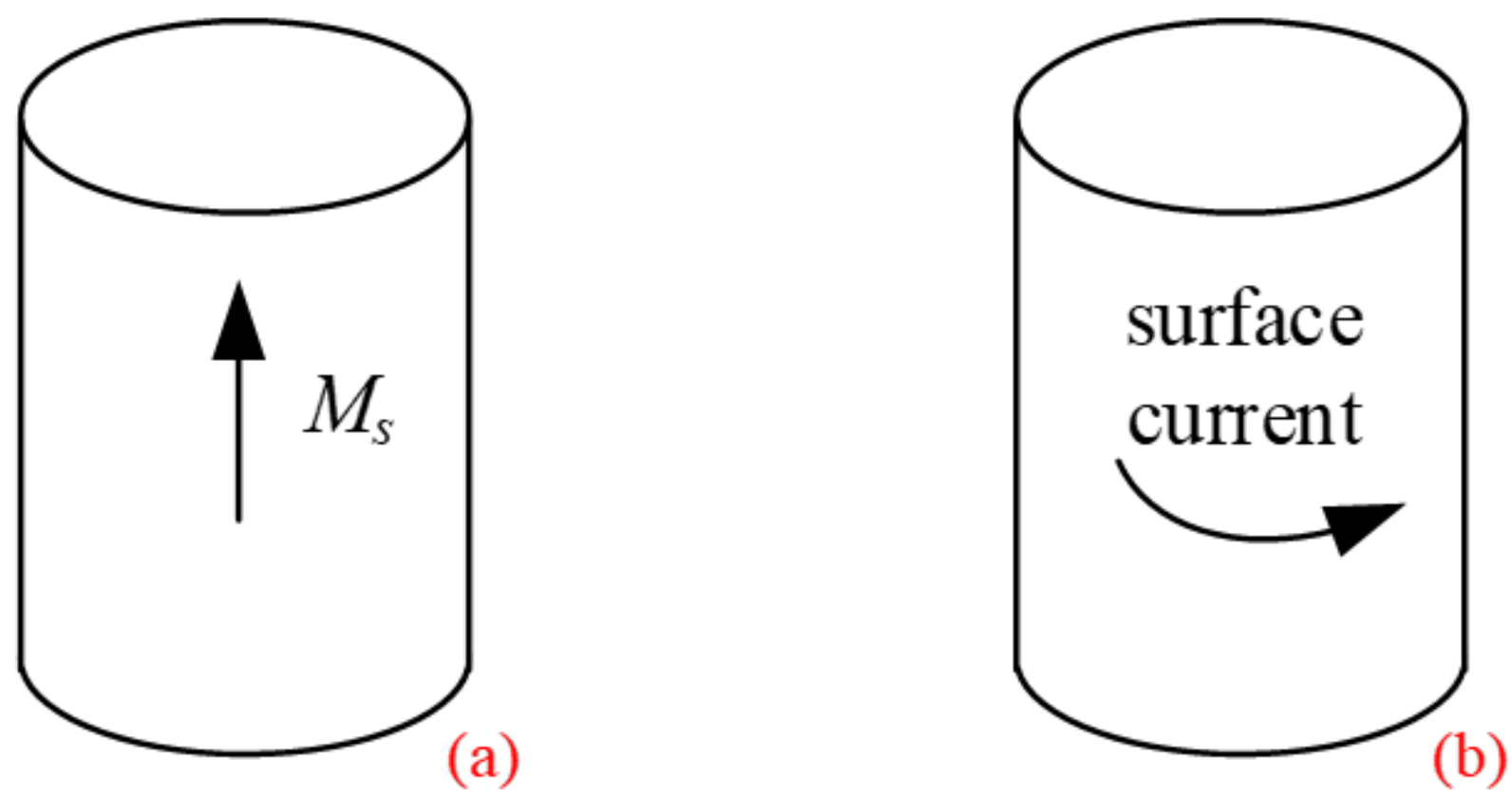

Figure 2

Cylindrical magnet, (a) polarized along its axis, (b) equivalent surface current. 

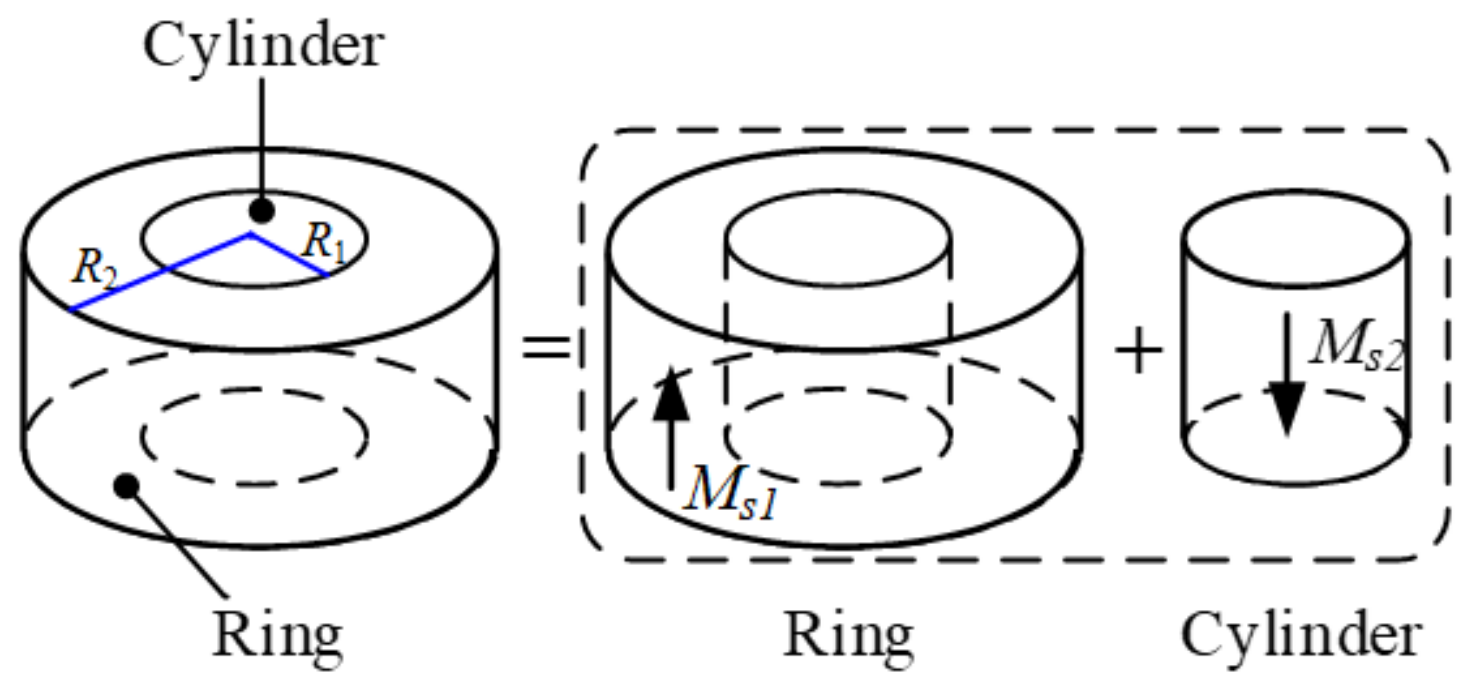

Figure 3

The array of permanent magnets structure.
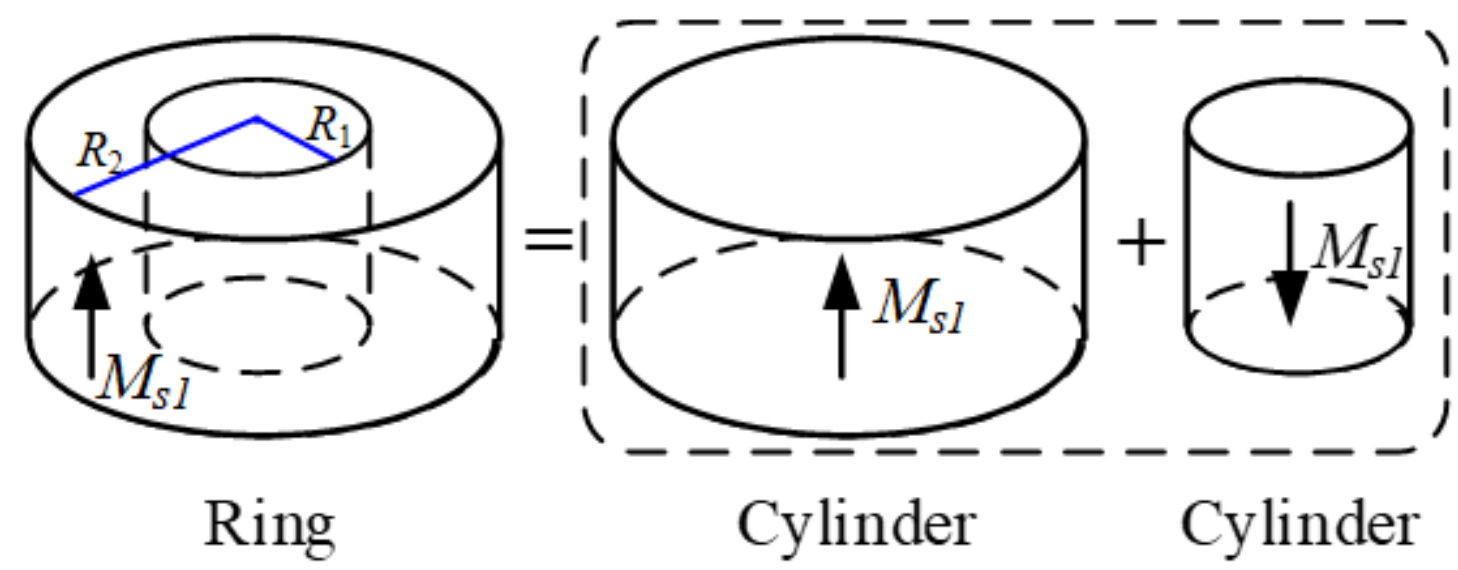

Figure 4

Ring-shaped magnet equivalence. 

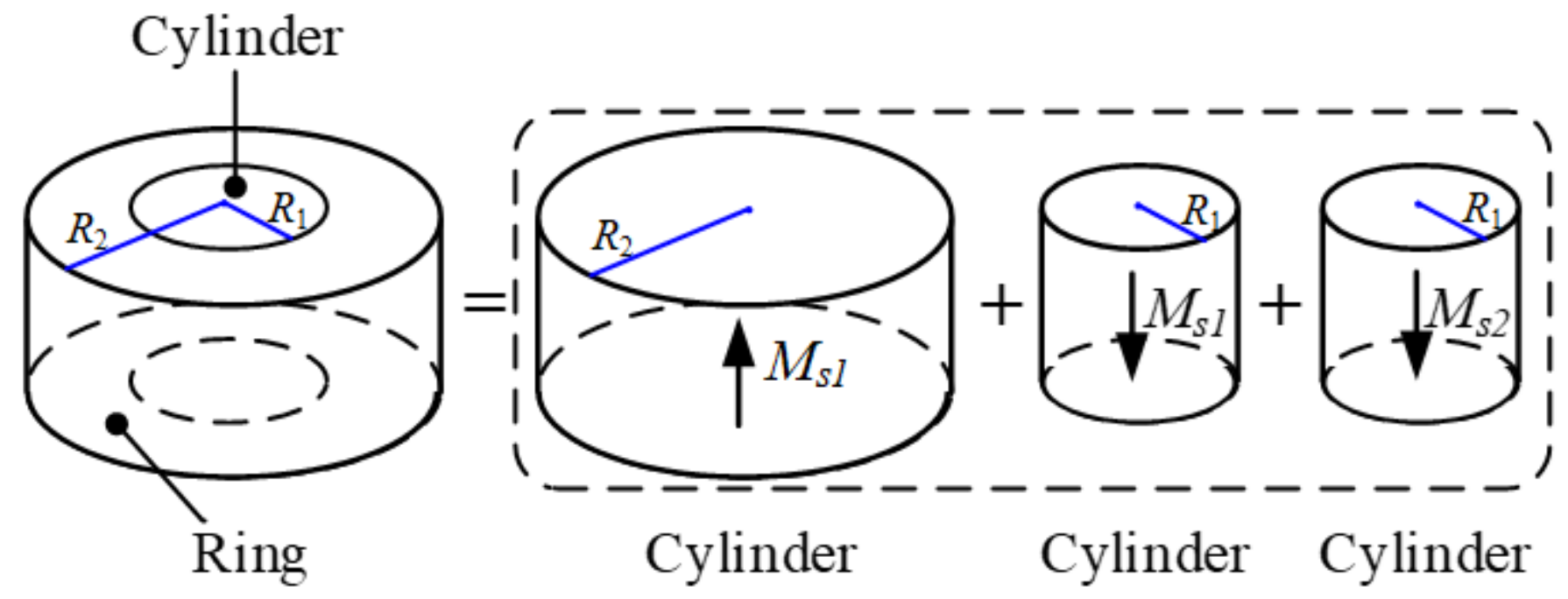

Figure 5

The array of permanent magnets equivalence.

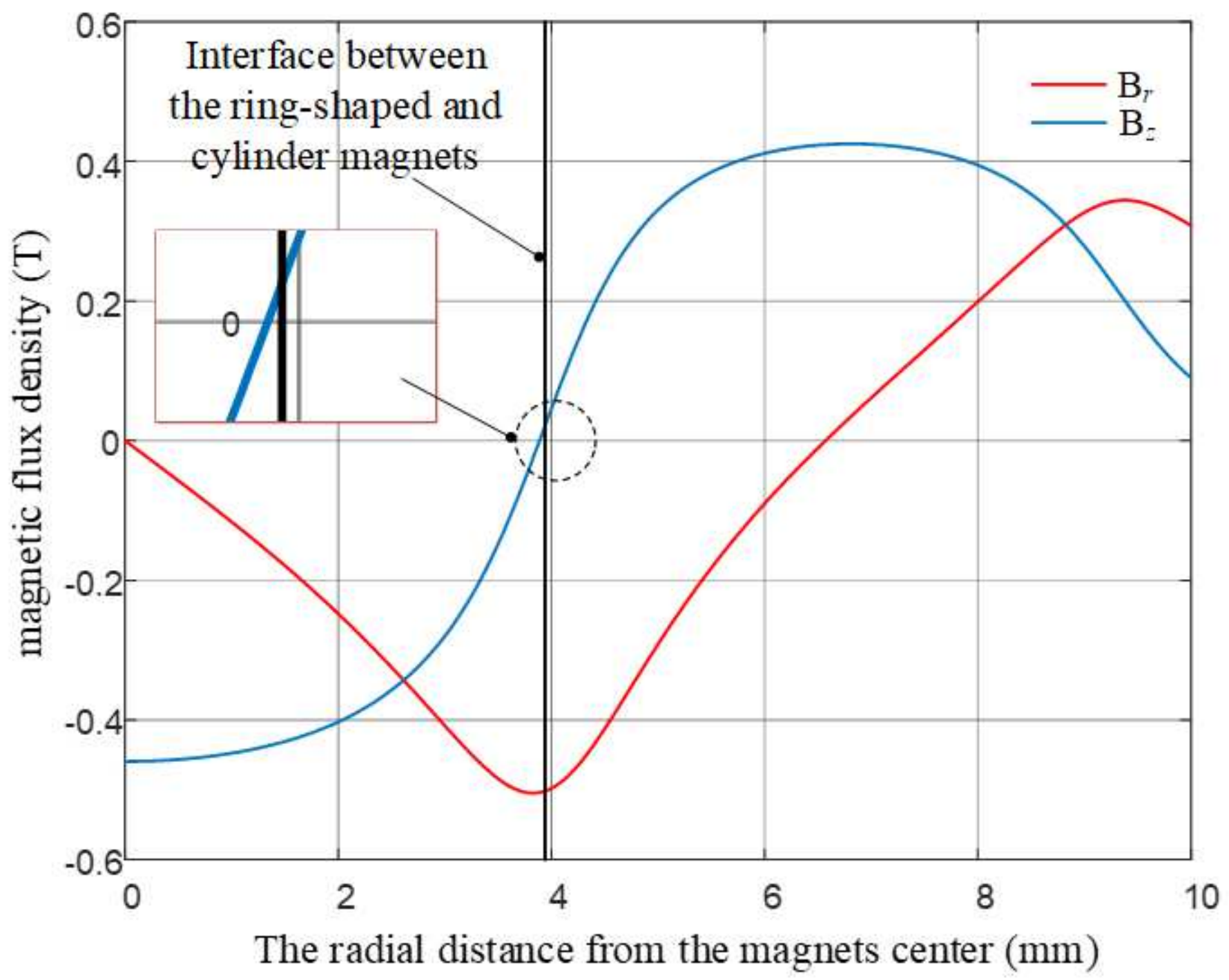


Figure 6

Theoretical magnetic flux density distribution in the plane parallel the top surface of permanent magnets wherein the distance between plane and top surface is $0.9 \mathrm{~mm}$.
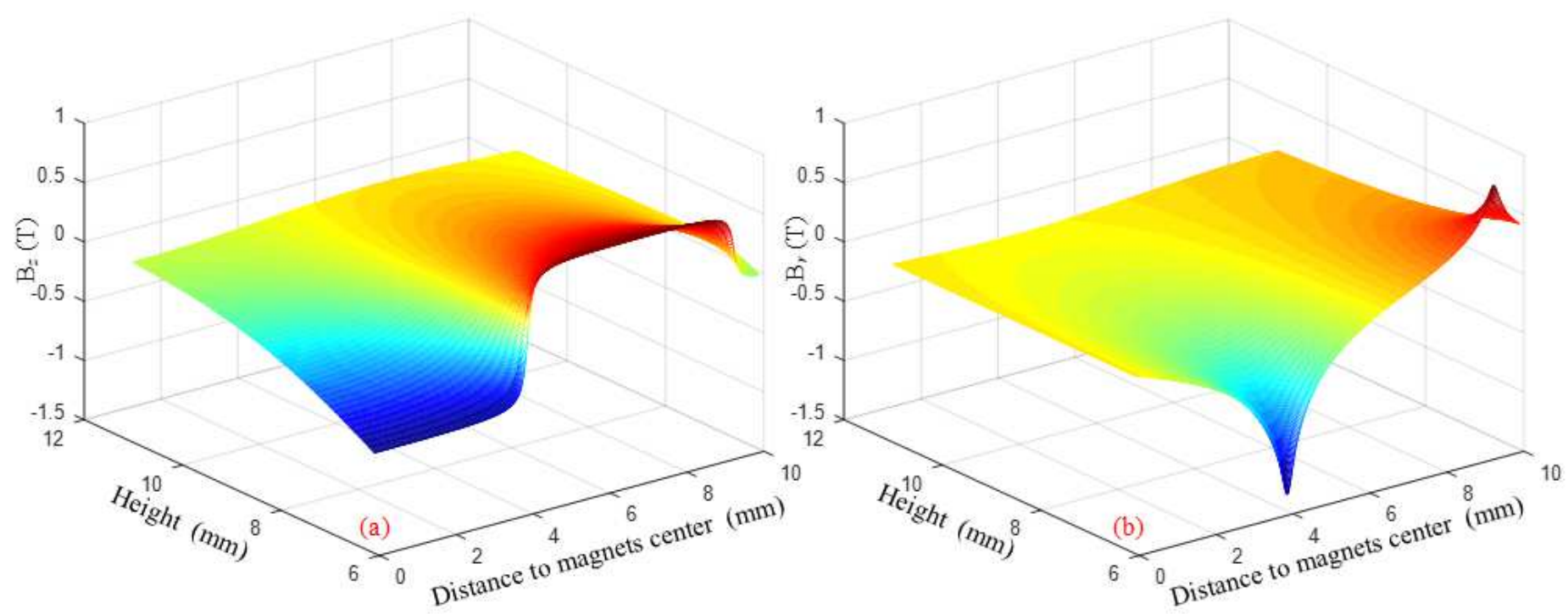

Figure 7

The height affection for the magnetic flux density generated by the array of permanent magnets, (a) Bz (b) $\mathrm{Br}$. 


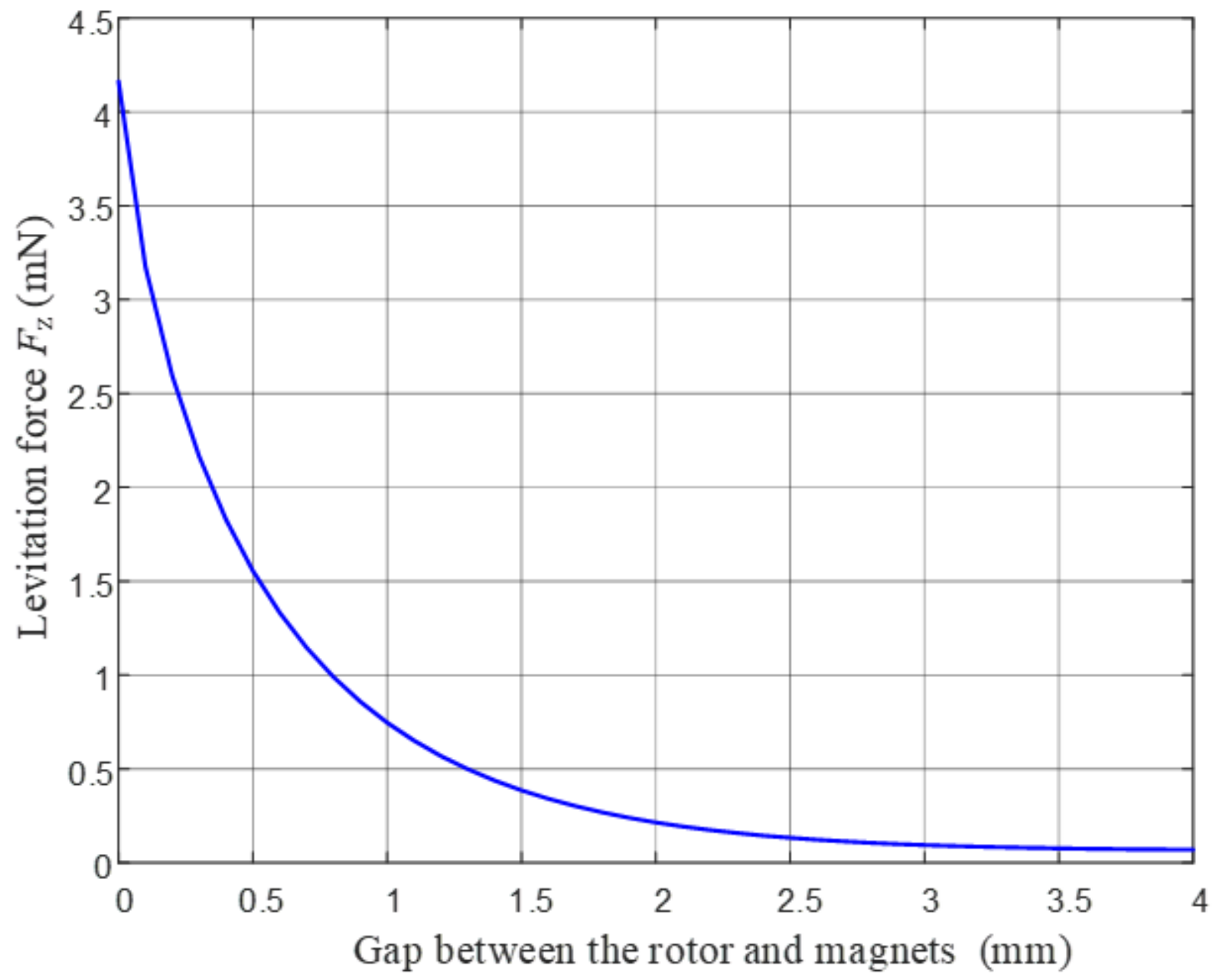

Figure 8

Theoretical diamagnetic levitation force versus levitation height. 


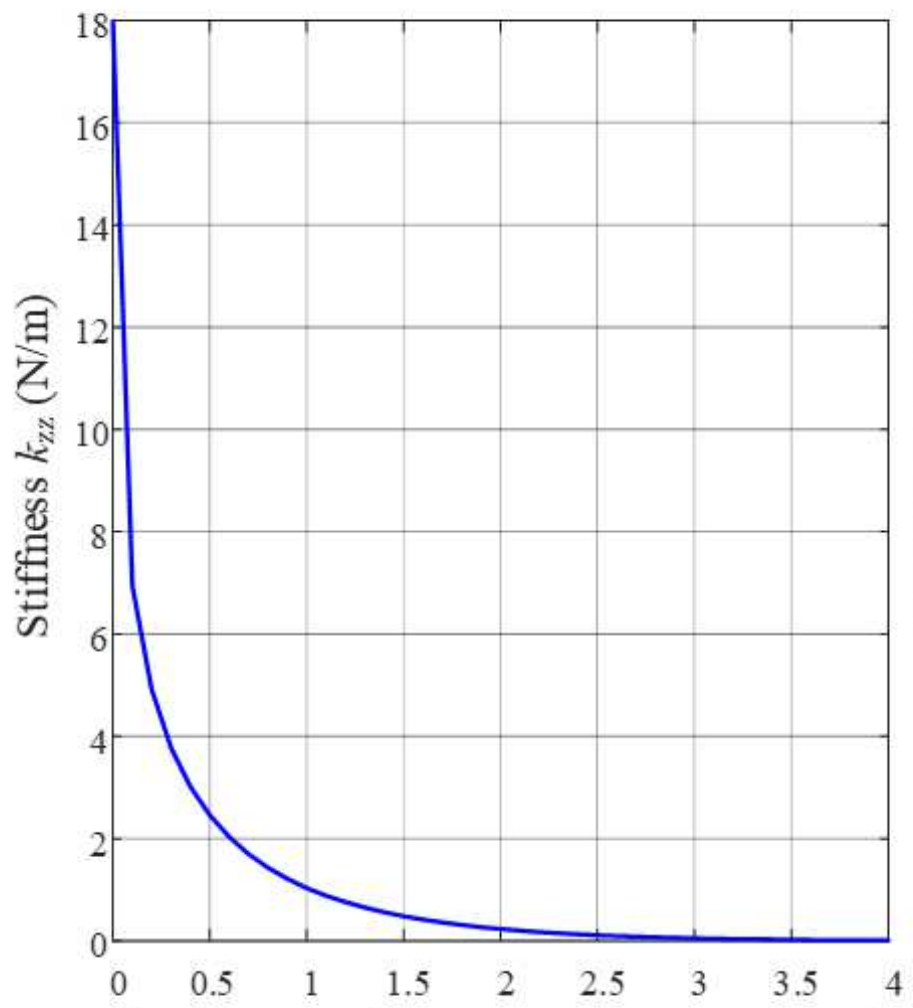

Gap between the rotor and magnets $(\mathrm{mm})$

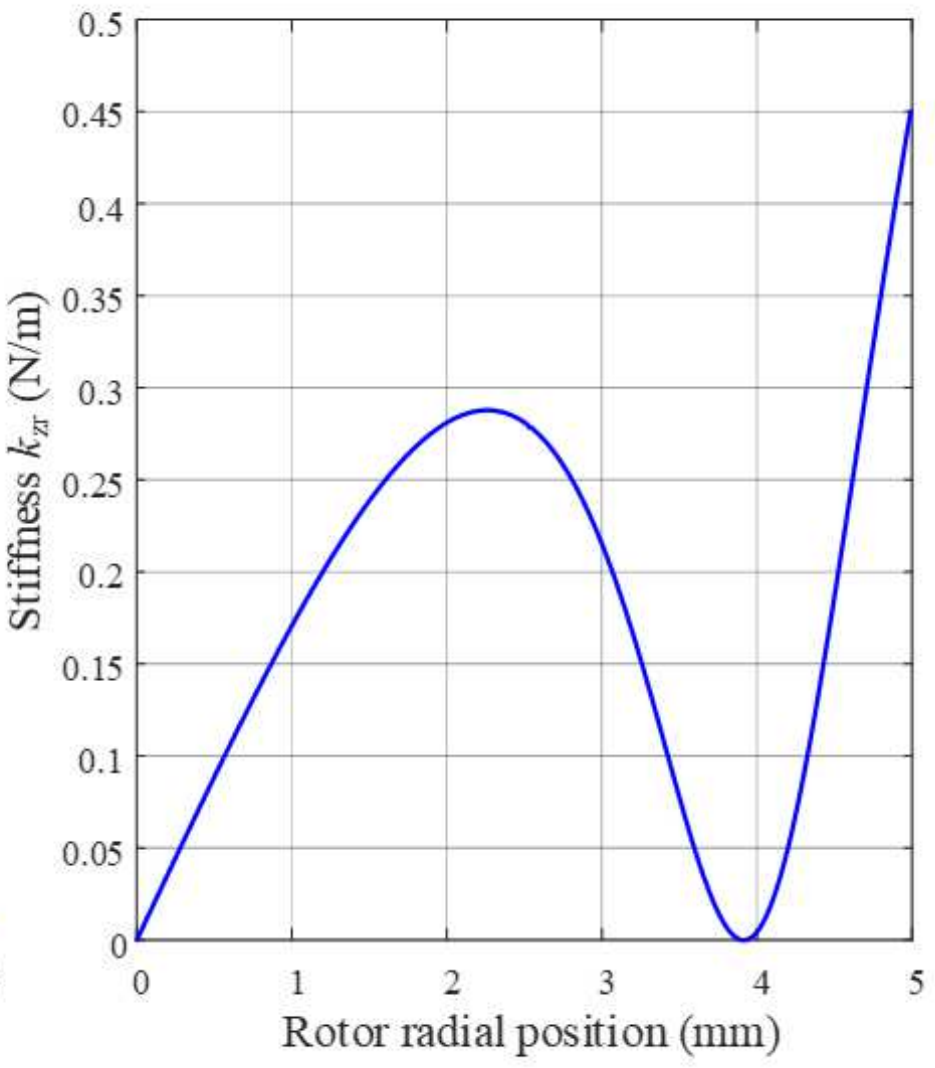

Figure 9

The stiffness property of the diamagnetic levitation force, (a) at different height, (b) at different rotor radial position with $0.9 \mathrm{~mm}$ height. 


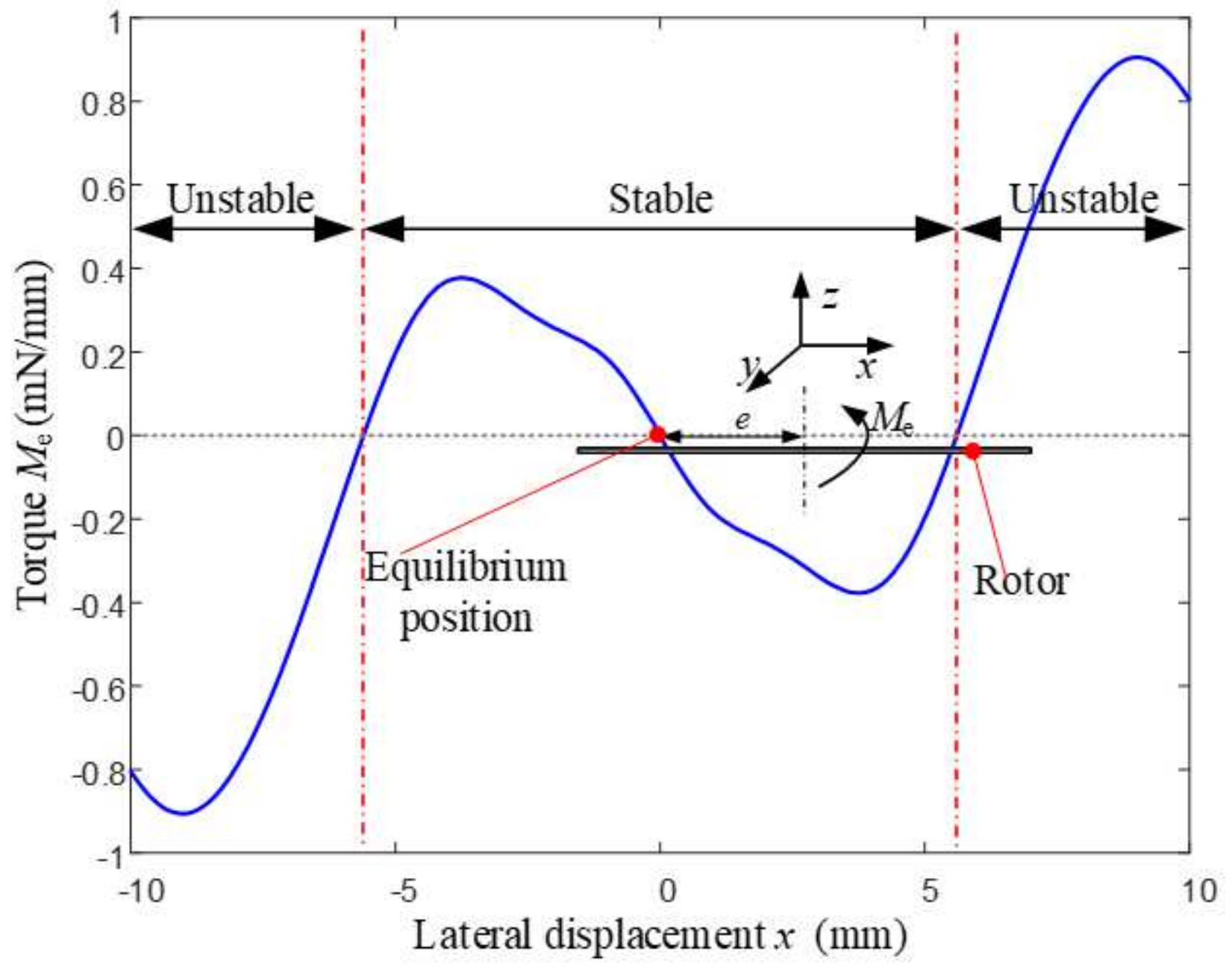

Figure 10

The torques applied to the rotor considering different lateral displacement (rotor radius is $5.19 \mathrm{~mm}$, levitation height is $0.9 \mathrm{~mm}$ ) 


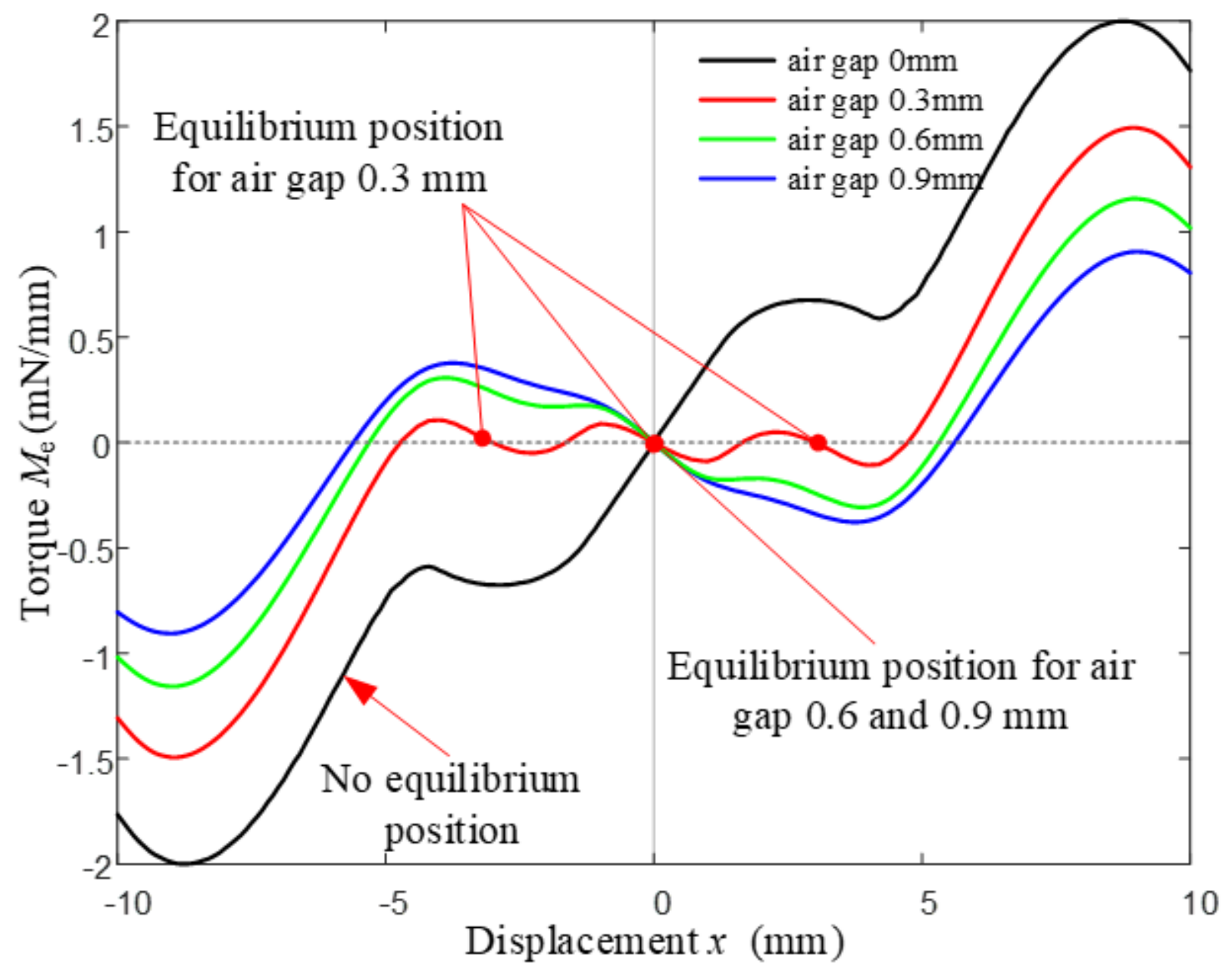

Figure 11

The lateral displacement of rotor versus torque under different levitation height of $0,0.3,0.6$ and $0.9 \mathrm{~mm}$ (rotor radius is $5.19 \mathrm{~mm}$ ) 


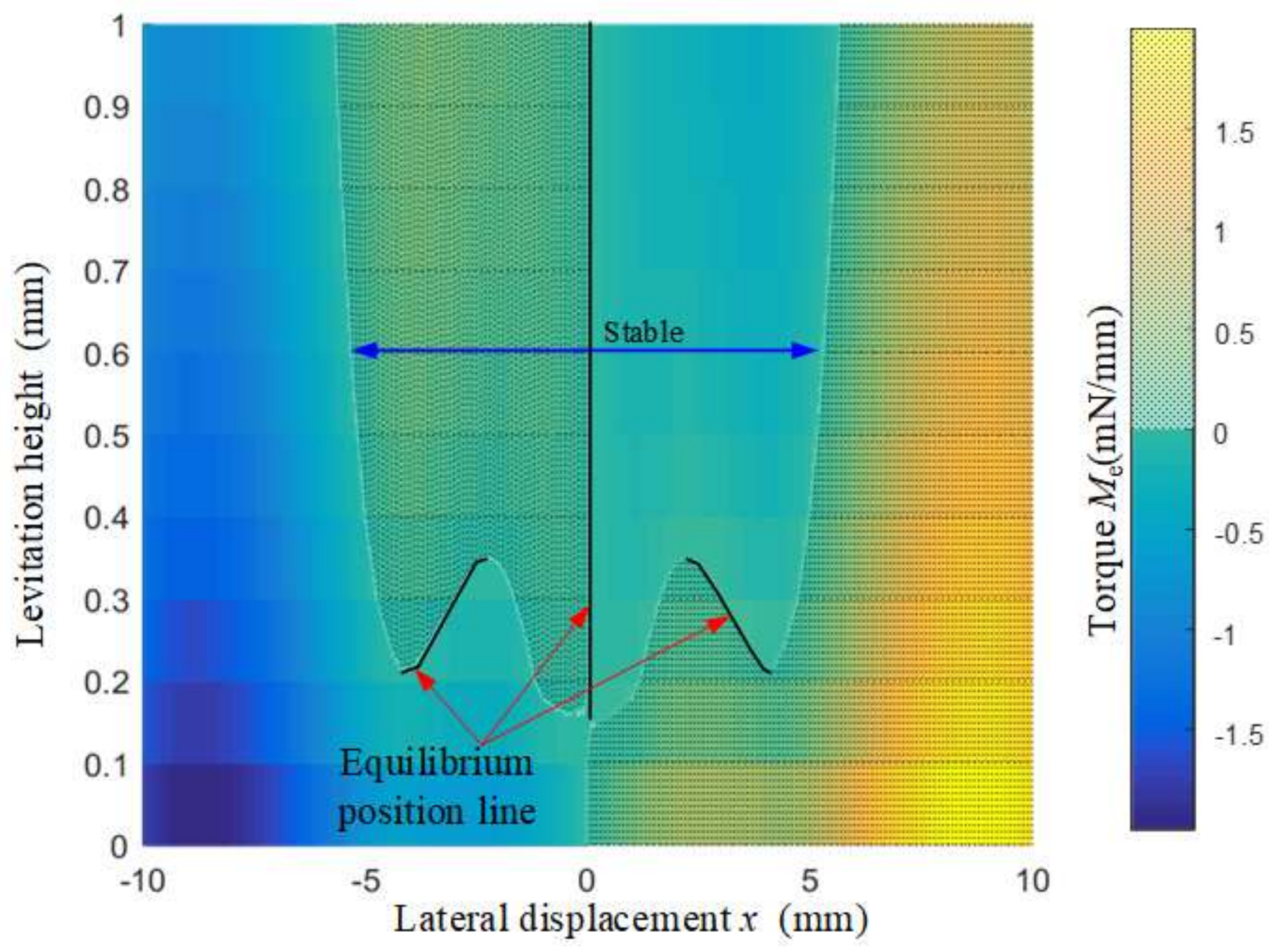

Figure 12

Torque of rotor versus levitation height and lateral displacement (rotor radius is $5.19 \mathrm{~mm}$ ). 


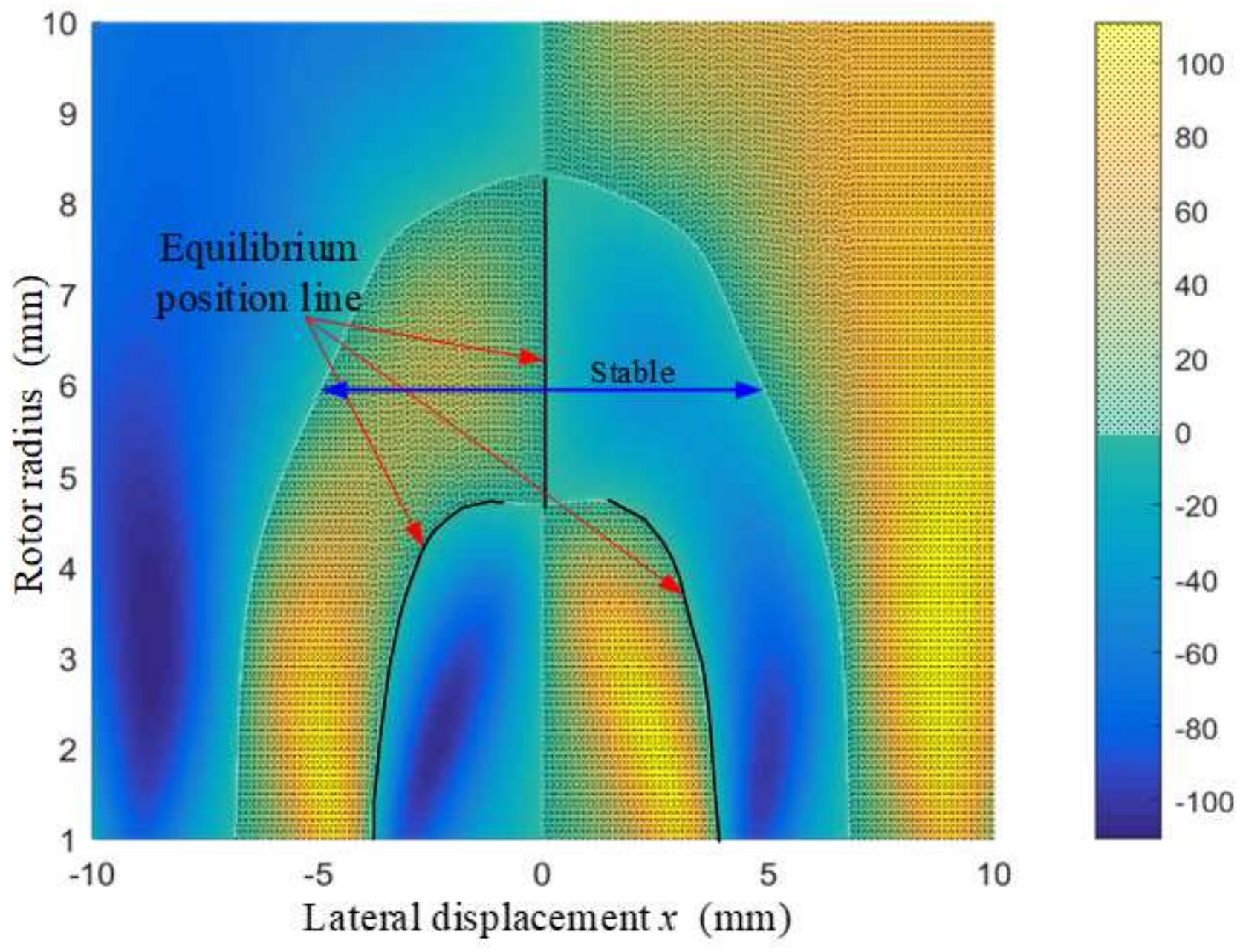

Figure 13

The torque of rotor versus rotor radius and rotor lateral displacement (levitation height $0.9 \mathrm{~mm}$ ).
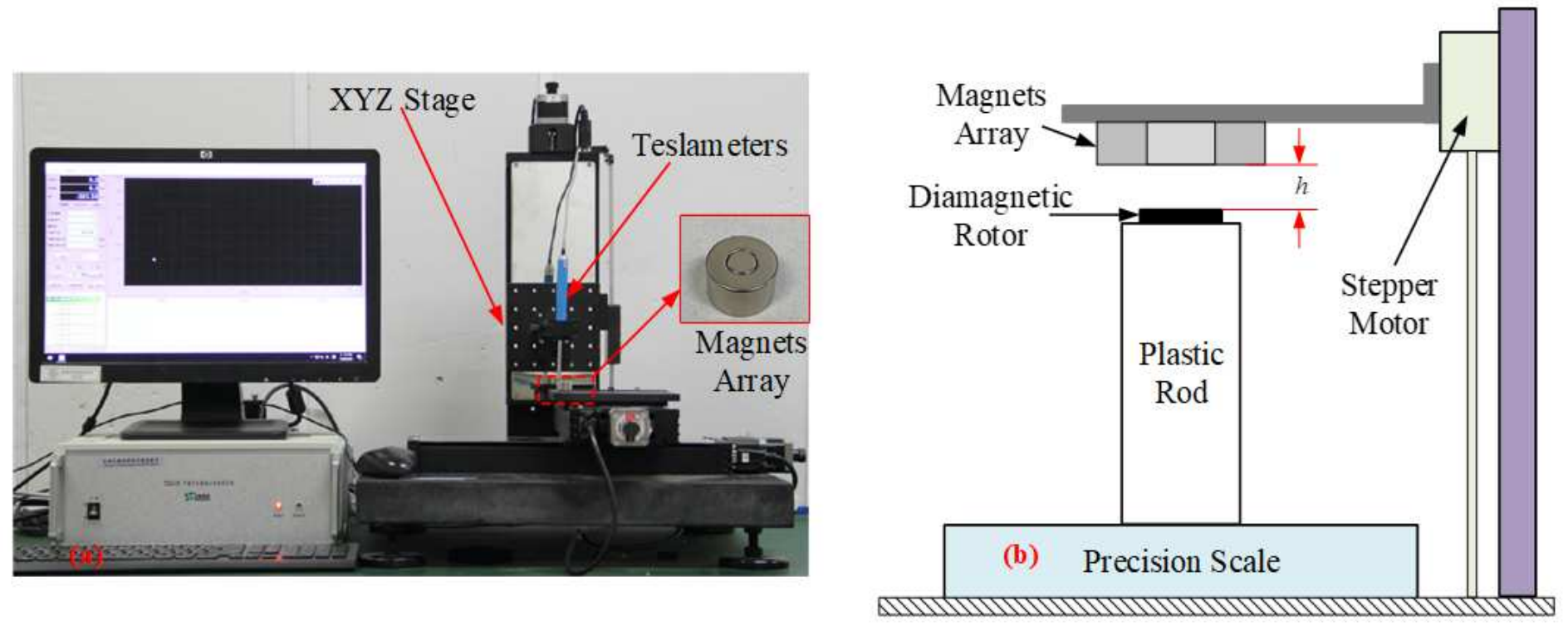

Figure 14 
Experimental setup, (a) magnetic field distribution tester, (b) diamagnetic levitation force measurement schematic diagram.
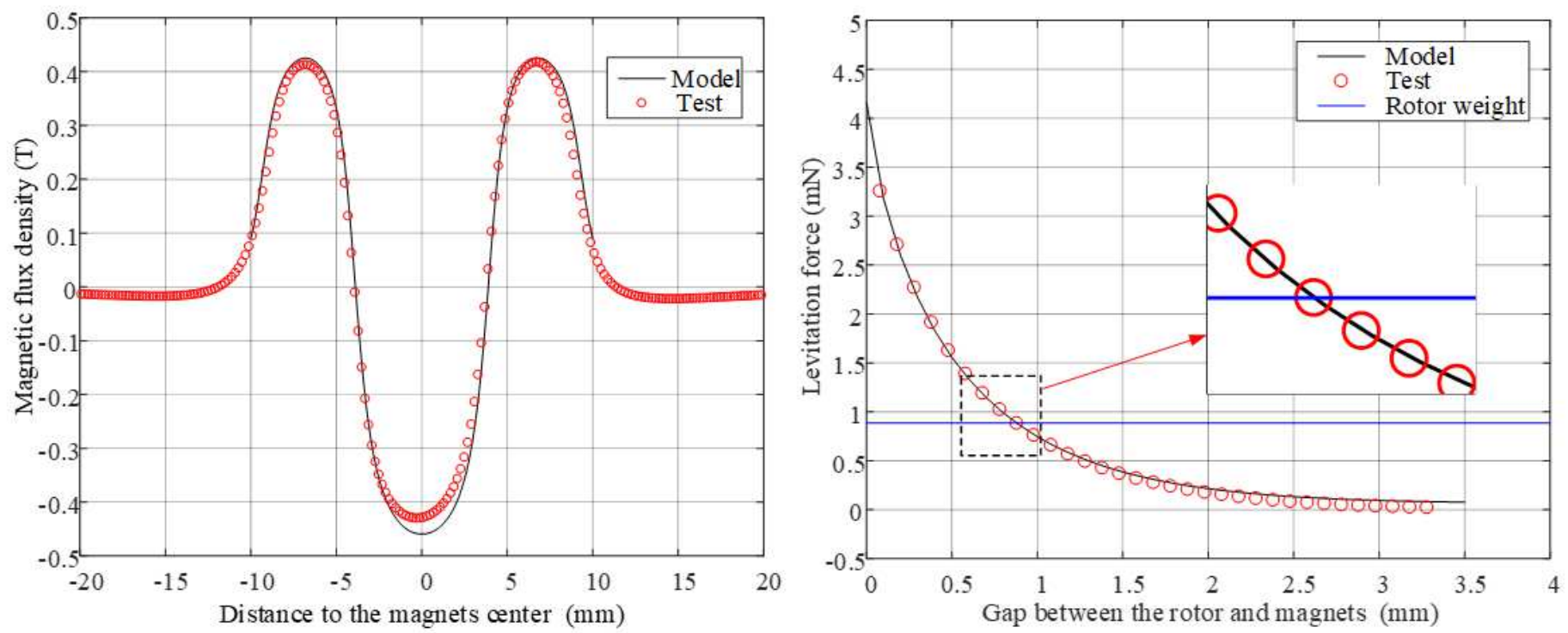

\section{Figure 15}

Comparison from the test and the model, (a) the magnetic flux density $\mathrm{Bz}$, (b) the diamagnetic levitation force.
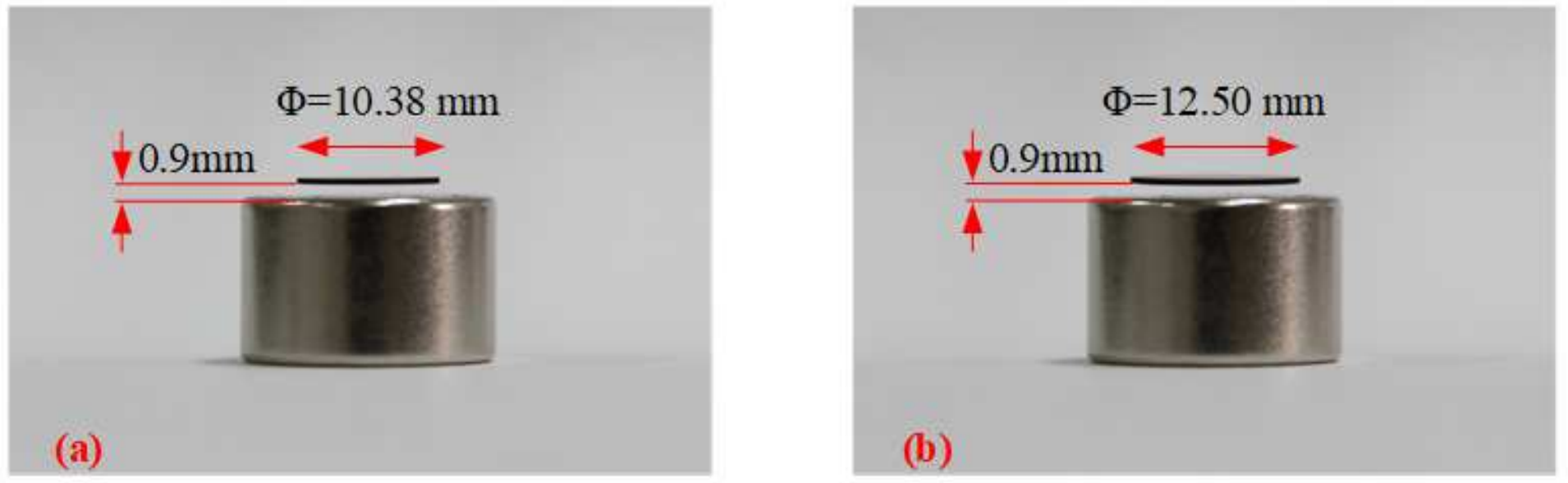

\section{Figure 16}

Levitation stability test, (a) radius $5.19 \mathrm{~mm}$, (b) radius $6.25 \mathrm{~mm}$. 

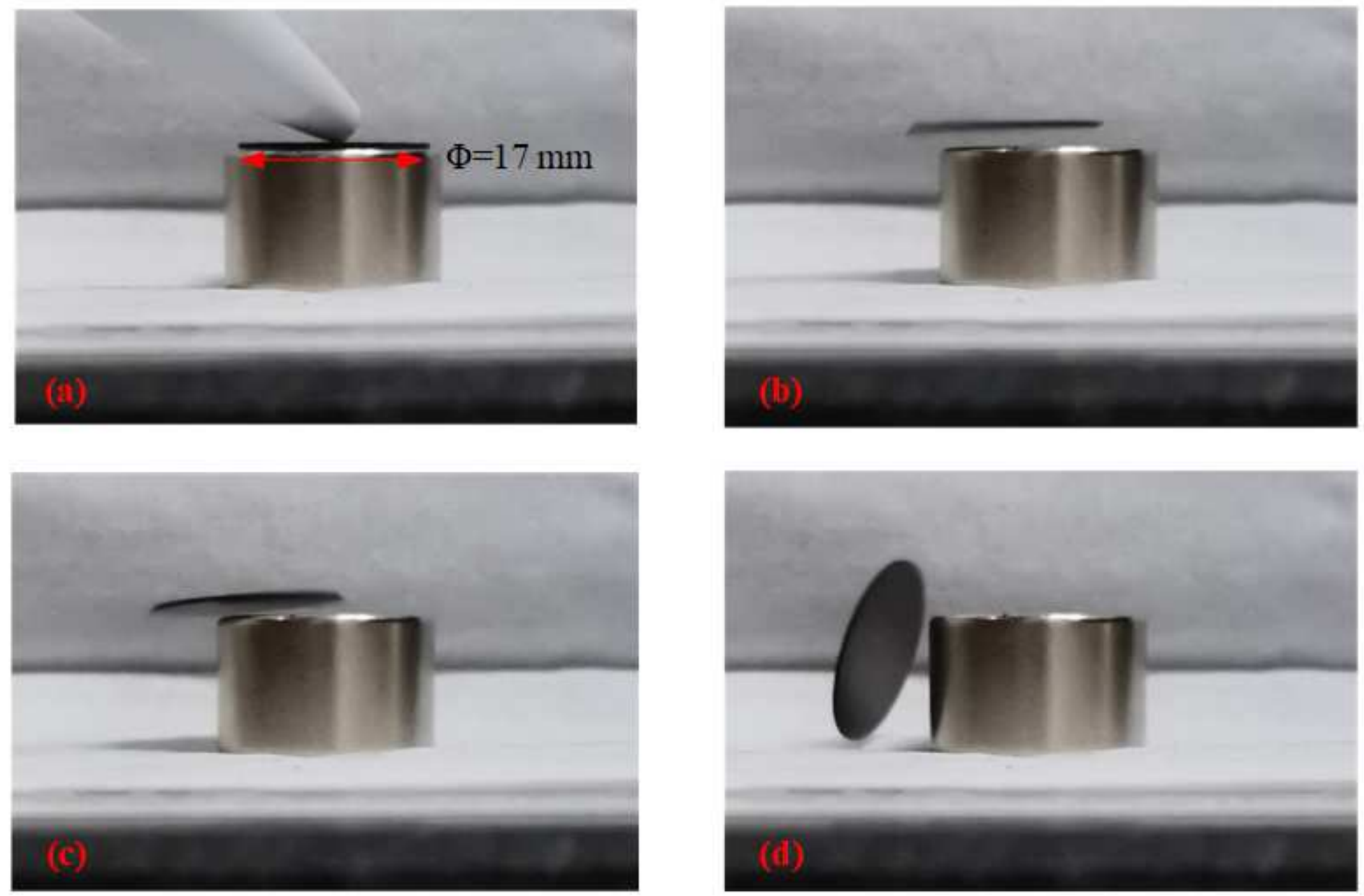

Figure 17

Levitation stability test for radius $8.50 \mathrm{~mm}$ rotor. 